\title{
THE APPLICATION OF A BOUNDARY INTEGRAL EQUATION METHOD TO THE PREDICTION OF DUCTED FAN ENGINE NOISE
}

\author{
M. H. Dunn and J. Tweed \\ Old Dominion University \\ Norfolk, VA \\ F. Farassat \\ NASA Langley Research Center \\ Hampton, VA
}




\begin{abstract}
The prediction of ducted fan engine noise using a boundary integral equation method (BIEM) is considered. Governing equations for the BIEM are based on linearized acoustics and describe the scattering of incident sound by a thin, finite-length cylindrical duct in the presence of a uniform axial inflow. A classical boundary value problem (BVP) is derived that includes an axisymmetric, locally reacting liner on the duct interior. Using potential theory, the BVP is recast as a system of hypersingular boundary integral equations with subsidiary conditions. We describe the integral equation derivation and solution procedure in detail. The development of the computationally efficient ducted fan noise prediction program TBIEM3D, which implements the BIEM, and its utility in conducting parametric noise reduction studies are discussed. Unlike prediction methods based on spinning mode eigenfunction expansions, the BIEM does not require the decomposition of the interior acoustic field into its radial and axial components which, for the liner case, avoids the solution of a difficult complex eigenvalue problem. Numerical spectral studies are presented to illustrate the nexus between the eigenfunction expansion representation and BIEM results. We demonstrate BIEM liner capability by examining radiation patterns for several cases of practical interest.
\end{abstract}




\section{OUTLINE}

$\S 1$. Introduction

$\S 2$. Boundary Value Problem (BVP) Derivation

2.1. Governing Differential Equations

2.2. Boundary Conditions

2.3. Far Field Radiation Condition and Edge Conditions

2.4. BVP Summary

$\S 3$. Boundary Integral Equation (BIE) Formulation

3.1. Helmholtz Potential Representation

3.2. Surface Operator Notation

3.3. Surface Kernel Properties

3.4. Layer Continuity Properties

3.5. Boundary Integral Equations

3.6. Edge Conditions

3.7. BIE Summary

$\S 4$. Integral Equation Solution

4.1. Hardwall Interior with No Inflow

4.2. Hardwall Interior with Inflow

4.3. Lined Interior with No Inflow

4.4. Lined Interior with Inflow

$\S 5$. Results

5.1. The Ducted Fan Noise Prediction Code TBIEM3D

5.2. Hard Wall Interior: Spectral Analysis

5.3. Sample Liner Calculations

$\S$ 6. Concluding Remarks

$\S 7$. References

$\S 8$. Appendices

8.1. Appendix A: Singular Kernel Analysis

8.2. Appendix B: Singular Integral Evaluations

\section{$\S 9$. Notation}

9.1. Parameters and Variables

9.2. Integral Equation Operators and Kernels 


\section{$\S 1$. INTRODUCTION}

Advanced analytical tools for predicting the sound radiated from engine ducts facilitate the design of active and passive noise abatement technology. To be useful in design studies, prediction tools should be fast, versatile, accurate, valid for a wide range of engineering situations, and implementable on mainstream computer systems. The ability to compute any portion of the sound field without the need to calculate the entire field is an important attribute in this regard. Conventional computational approaches such as Finite Element Methods, CFD, and Computational Aeroacoustics (CAA) lack this property which limits their usefulness for conducting parametric noise studies. On the other hand, boundary integral or boundary element prediction methods calculate the acoustic field pointwise allowing the designer to compute noise only at acoustically sensitive regions of space.

In this paper, we present a boundary integral equation method (BIEM) for predicting ducted fan engine noise. The BIEM is based on the equations of linearized acoustics with uniform inflow and predicts the sound scattered by an infinitesimally thin, finite length cylindrical duct that has been irradiated by some simple source process. Boundary conditions on the duct interior allow for an axisymmetric, axially segmented, locally reactive liner with circumferentially uniform impedance. The liner can be positioned anywhere inside the duct (see figure 1). The special case of a hard wall interior was considered in reference [1].

Simple acoustic sources, such as point or line monopoles and dipoles, are used to generate incident sound. Source configurations are composed of $N$ symmetrically spaced (circumferentially) line or point sources and are situated on a disc perpendicular to the duct axis (see figure 1). If the source strengths are constant in time and the source disk spins with uniform angular speed $\widetilde{\Omega}$, then classical spinning modes appear inside the duct. Spinning modes can also 
be generated by nonrotating sources with time harmonic strengths. By suitable choices of monopole and dipole strengths, rotating source configurations can be constructed to simulate the loading and thickness components of fan noise.

In the early 1960's, Tyler and Sofrin first introduced the concept of applying linear infinite duct, spinning mode analysis to the prediction of ducted fan engine noise [2]. In this pioneering research, simple propagation and radiation models were applied to single radial modes incident on the duct inlet. Reflection and inflow effects were ignored and only hard wall boundary conditions were considered. Many investigators have since expanded these concepts to include enhanced radiation models, inflow effects, and liner capability. The volume of research on this subject is considerable and will not be reviewed in any detail here. Comprehensive surveys can be found in references [3-5].

The finite duct BIEM presented here provides two fundamental improvements over simple infinite duct prediction methods. First, the coupling of radiation and propagation for the infinite duct methods requires an estimate of the generalized impedances at the inlet and exhaust planes. These quantities are then used as input to some radiation model, such as Rayleigh's formula for sound radiation from a flanged cylinder, to calculate the acoustic farfield. For the finite duct BIEM, knowledge of the generalized impedances is not required. Reflections at the duct openings are determined implicitly. Furthermore, unlike Rayleigh's formula, the BIEM accounts for edge diffraction and permits noise predictions in the duct shadow region. Second, infinite duct methods require the solution of an eigenvalue problem in which the internal acoustic field is written as a linear combination of circumferential, radial, and axial eigenfunctions. For the liner case, the eigenvalue problem is difficult to solve due to the appearance of Bessel functions with complex argument. A body of research, which is reviewed in reference [3], has been devoted to 
the solution of this very difficult eigenvalue problem. The BIEM does not involve the solution of the eigenvalue problem because only a circumferential decomposition of the acoustic field is required. If desired, the radial and axial content of the computed BIEM interior field can be obtained using Hankel and Fourier transform techniques.

Application of the BIEM to ducted fan noise prediction is a four step process which we summarize below:

Step 1) In accordance with the preceding assumptions we derive a classical mixed boundary value problem (BVP) for the scattered acoustic pressure..

Step 2) Using single and double layer Helmholtz potentials, the BVP is converted to a system of hypersingular integral equations for the unknown layer densities.

Step 3) The system of integral equations is solved numerically by the method of collocation in which the layer densities are approximated by finite series of orthogonal polynomials.

Step 4) The scattered sound field is computed pointwise by numerically integrating the Helmholtz potential representation.

Theoretical details of the BIEM form the bulk of this paper. Much analysis is devoted to the integral equation development and solution technique. The authors contend that innovations in these areas have yielded enhanced numerical procedures that simplify calculations and lead to rapid noise predictions. A ducted fan noise prediction program, TBIEM3D [6], that implements the BIEM has been developed. Computational results are presented to illustrate TBIEM3D noise prediction capabilities and to compare with eigenfunction expansion prediction methods. 


\section{$\S 2$. BOUNDARY VALUE PROBLEM DERIVATION}

We consider the scattering of sound by an infinitesimally thin, finite length cylindrical duct in the presence of a uniform axial inflow with Mach number $M$. The duct is irradiated by incident sound produced by a collection of $N$ simple point or line monopoles and/or dipoles. Acoustic propagation and radiation are based on the assumption of linearity. The $N$ sources have equal strengths, are located on a disk perpendicular to the duct axis, and are arranged symmetrically about the disk (refer to figure 1). The source disk is centered on the duct axis and either spins or is stationary. Source strengths are chosen so that the incident acoustic field can be written as a superposition of time harmonic, circumferential modes.

In the analysis that follows, all quantities are nondimensional: length by $\tilde{r}_{D}$, mass by $\tilde{\rho}_{0} \tilde{r}_{D}^{3}$, and time by $\frac{\tilde{r}_{D}}{\tilde{c}}$.

\subsection{GOVERNING DIFFERENTIAL EQUATIONS}

We adopt the point of view that the duct is translating in the axial $(+z)$ direction with uniform speed $\widetilde{U}$ and initially consider an Eulerian description of the acoustic field. The total acoustic pressure in the sound field is split into known incident and unknown scattered parts

$$
p^{\prime}(r, \psi, z, t)=p_{i}^{\prime}(r, \psi, z, t)+p_{s}^{\prime}(r, \psi, z, t) .
$$

In regions of space and time that contain no scattering surfaces, $p_{s}^{\prime}$ is governed by the homogeneous wave equation

$$
\left[\frac{\partial^{2}}{\partial t^{2}}-\frac{1}{r} \frac{\partial}{\partial r}\left(r \frac{\partial}{\partial r}\right)-\frac{1}{r^{2}} \frac{\partial^{2}}{\partial \psi^{2}}-\frac{\partial^{2}}{\partial z^{2}}\right] p_{s}^{\prime}=0 .
$$

Acoustic pressure and radial velocity are related through the radial component of the acoustic momentum equation 


$$
\frac{\partial u_{r}^{\prime}}{\partial t}+\frac{\partial p^{\prime}}{\partial r}=0
$$

In a frame of reference moving with the duct, the symmetry of the source process is such that all dependent acoustic variables can be expressed as linear superpositions of time harmonic circumferential modes. For example, the scattered pressure has the form

$$
p_{s}^{\prime}(r, \psi, Z, t)=\sum_{n=-\infty}^{\infty} P_{s}^{n}(r, Z) e^{i(k t-n N \psi)}
$$

and the radial component of total acoustic velocity is written

$$
u_{r}^{\prime}(r, \psi, Z, t)=\sum_{n=-\infty}^{\infty} U_{r}^{n}(r, Z) e^{i(k t-n N \psi)}
$$

where the stretched, moving axial coordinate $Z$ is given by

$$
Z=\frac{z-M t}{\beta} \text {. }
$$

Incident and total acoustic pressures are written similarly. Modal amplitudes in the BIEM are calculated term by term. For notational convenience, we drop the superscript $n$ on the modal coefficients and define the circumferential mode number $m$ by the equation $m=n N$.

If the source disk rotates with angular speed $\tilde{\Omega}$ and the strengths of the $N$ sources are time independent in the moving frame, then $k=\frac{m \tilde{\Omega} \tilde{r}_{D}}{\tilde{c}}$ and equations (4a-b) yield the classical spinning mode representations. In this case, the incident field can be made to simulate that produced by an $N$-bladed fan. This is accomplished by manipulating monopole and dipole strengths to approximate the loading and thickness components of fan noise. For non-spinning sources whose strengths are time harmonic with excitation frequency $\tilde{\omega}$, we have $k=\frac{\tilde{\omega} \tilde{r}_{D}}{\tilde{c}}$ and the time harmonic factor in $(4 a-b)$ can be removed from the summations. 
Rather than work with the customary convected wave equation, we simplify the governing equations by defining new dependent variables $Q, Q_{s}, Q_{i}$, and $V_{r}$ by the relations

$$
\begin{gathered}
Q(r, Z)=P(r, Z) e^{i \kappa M Z} \\
Q_{s}(r, Z)=P_{s}(r, Z) e^{i \kappa M Z} \\
Q_{i}(r, Z)=P_{i}(r, Z) e^{i \kappa M Z} \\
V_{r}(r, Z)=U_{r}(r, Z) e^{i \kappa M Z}
\end{gathered}
$$

Combining (4-6) with (2) yields the two dimensional Helmholtz equation

$$
\left[\frac{1}{r} \frac{\partial}{\partial r}\left(r \frac{\partial}{\partial r}\right)+\frac{\partial^{2}}{\partial Z^{2}}-\frac{m^{2}}{r^{2}}+\kappa^{2}\right] Q_{s}=0
$$

for the $m$-th modal coefficient of scattered pressure. Using (3-6), the $m$-th radial component of the momentum equation (3) can be written as

$$
e^{-i \frac{\kappa}{M} Z} V_{r}(r, Z)=\frac{\beta}{M} \int_{-\infty}^{Z} e^{-i \frac{\kappa}{M} Z^{\prime}} \frac{\partial Q}{\partial r}\left(r, Z^{\prime}\right) d Z^{\prime} .
$$

If the duct is stationary, then (7) and (8) reduce to

$$
\left[\frac{1}{r} \frac{\partial}{\partial r}\left(r \frac{\partial}{\partial r}\right)+\frac{\partial^{2}}{\partial Z^{2}}-\frac{m^{2}}{r^{2}}+k^{2}\right] P_{s}=0
$$

and

$$
U_{r}(r, Z)=\frac{i}{k} \frac{\partial P}{\partial r}(r, Z) .
$$

We note that (7-10) are valid for points not lying on the stretched duct.

\subsection{BOUNDARY CONDITIONS}

To meet noise certification requirements, it is necessary to treat the engine duct with noise suppression devices. We consider an axisymmetric, locally reactive liner on the duct interior wall whose surface admittance is circumferentially uniform. The admittance in the axial direction is assumed to be piecewise constant. This property implies that any portion of the duct interior may 
be lined or hard and allows for inclusion of an axially segmented liner. The hardwall boundary condition is used for the duct exterior wall.

We introduce the concept of a surface function to facilitate discussions of the boundary condition and subsequent integral equation derivations. Let $f(r, Z)$ be an arbitrary field function and assume that the stretched, moving axial coordinates of the duct trailing and leading edges are $a$ and $b$ respectively. Define the surface functions $f^{ \pm}(Z)$ by the formulas

$$
f^{ \pm}(Z)=\lim _{r \rightarrow l^{ \pm}} f(r, Z) \quad Z \in(a, b) .
$$

Note that the positive (negative) superscript refers to the duct exterior (interior) surface.

On the outer duct wall the hardwall boundary condition implies that

$$
V_{r}^{+}(Z)=0 \quad Z \in(a, b) .
$$

In reference [7] it is shown that if $\alpha$ represents the circumferentially uniform and axially piecewise constant specific acoustic admittance on the duct interior surface, then in the stretched, moving frame of reference, the surface modal coefficients of radial velocity and pressure satisfy the boundary equation

$$
-e^{-i \frac{\kappa}{M} Z} V_{r}^{-}(Z)+\frac{i M \alpha}{\beta^{2} \kappa} \frac{d}{d Z}\left(e^{-i \frac{\kappa}{M} Z} Q^{-}\right)(Z)=0 \quad Z \in(a, b) .
$$

In the absence of flow, the boundary conditions reduce to

$$
U_{r}^{+}(Z)=0 \quad Z \in(a, b)
$$

and

$$
-U_{r}^{-}(Z)+\alpha P^{-}(Z)=0 \quad Z \in(a, b) .
$$

The momentum equation (8) is used to eliminate the radial velocity from (12a-b) yielding

$$
\begin{aligned}
& \left(\frac{\partial Q}{\partial r}\right)^{+}(Z)=0 \quad Z \in(a, b) \\
& -e^{-i \frac{\kappa}{M} Z}\left(\frac{\partial Q}{\partial r}\right)^{-}(Z)+\frac{i M^{2} \alpha}{\beta^{3} \kappa} \frac{d^{2}}{d Z^{2}}\left(e^{-i \frac{\kappa}{M} Z} Q^{-}\right)(Z)=0 \quad Z \in(a, b),
\end{aligned}
$$


and

$$
\int_{-\infty}^{a} e^{-i \frac{\kappa}{M} Z^{\prime}} \frac{\partial Q}{\partial r}\left(1, Z^{\prime}\right) d Z^{\prime}=0
$$

Equations (14a-b) are obtained by differentiating (12a-b) and (15) results by evaluating (12a) at the trailing edge. These equations are valid for $0 \leq M<1$ but (15) is satisfied trivially for $M \rightarrow 0$ and provides no additional information. Note that for $M=0$ and $\alpha=0$ (hardwall interior) we have the classical Neumann boundary conditions. Also, if we add a second coannular duct, then boundary conditions similar to (14-15) also apply to the second duct.

\subsection{FARFIELD RADIATION CONDITION AND EDGE CONDITIONS}

In order to have a uniquely solvable BVP we must constrain the behavior of the acoustic pressure in the farfield and at the duct edges. To ensure continuity of velocity at the trailing edge we impose the Kutta condition

$$
\lim _{Z \rightarrow a^{+}}\left[Q^{+}(Z)-Q^{-}(Z)\right]=0
$$

For physically reasonable solutions to exist we also require the acoustic pressure to be integrable everywhere and particularly in any neighborhood about the leading edge.

The boundary value problem is finalized by applying the Sommerfeld farfield radiation condition, yielding the constraint

$$
\lim _{\rho=\sqrt{r^{2}+Z^{2}} \rightarrow \infty} \sqrt{\rho}\left(\frac{\partial Q}{\partial \rho}+i \kappa Q\right)=0
$$

\subsection{BVP SUMMARY}

We summarize the above results by listing a complete two-dimensional BVP for the unknown scattered acoustic pressure in terms of the known incident pressure. It is assumed that the 
functions $Q_{i}$ and its derivatives are known, satisfy (17), and are continuous across duct surface.

For $M \geq 0$

$$
\begin{aligned}
& {\left[\frac{1}{r} \frac{\partial}{\partial r}\left(r \frac{\partial}{\partial r}\right)+\frac{\partial^{2}}{\partial Z^{2}}-\frac{m^{2}}{r^{2}}+\kappa^{2}\right] Q_{s}=0 \quad(r, Z) \text { not on duct surface }} \\
& Q(r, Z)=Q_{i}(r, Z)+Q_{s}(r, Z) \quad \forall(r, Z) \\
& \left(\frac{\partial Q}{\partial r}\right)^{+}(Z)=0 \quad Z \in(a, b) \\
& -e^{-i \frac{\kappa}{M} Z}\left(\frac{\partial Q}{\partial r}\right)^{-}(Z)+\frac{i M^{2} \alpha}{\beta^{3} \kappa} \frac{d^{2}}{d Z^{2}}\left(e^{-i \frac{\kappa}{M} Z} Q^{-}\right)(Z)=0 \quad Z \in(a, b) \\
& \int_{-\infty}^{a} e^{-i \frac{\kappa}{M} Z^{\prime}} \frac{\partial Q}{\partial r}\left(1, Z^{\prime}\right) d Z^{\prime}=0 \\
& \lim _{Z \rightarrow a^{+}}\left[Q_{s}^{+}(Z)-Q_{s}^{-}(Z)\right]=0 \\
& \int_{\Re}\left|Q_{s}\right| d \Re<\infty \quad \Re \text { any neighborhood about leading edge } \\
& \lim _{\rho=\sqrt{r^{2}+Z^{2}} \rightarrow \infty} \sqrt{\rho}\left(\frac{\partial Q_{s}}{\partial \rho}+i \kappa Q_{s}\right)=0
\end{aligned}
$$

\section{§ 3. THE BOUNDARY INTEGRAL EQUATION FORMULATION}

In this section, we convert the two-dimensional boundary value problem (18a-h) to a system of boundary integral equations using Helmholtz potentials [8]. This approach is motivated by its computational simplicity relative to purely numerical methods based on finite differences or finite elements.

These latter methods involve the calculation and storage of the entire acoustic field and must resort to special treatment at the farfield boundary. Consequently, high frequency, farfield 
predictions require excessive computer memory and computational time. It is shown in reference [9] that these drawbacks can be diminished somewhat by coupling nearfield finite-element results to Kirchhoff's radiation formula.

Potential methods, as applied here, involve the pointwise calculation of the acoustic field by evaluating duct surface integrals. Noise predictions are calculated at user defined locations only and computer storage is minimal. The potential representation satisfies the Sommerfeld radiation condition implicitly. Thus, numerical treatment at a fictitious farfield boundary is avoided. Input to the potential integrals is obtained by solving a system of hypersingular integral equations for unknown surface functions. The difficulties associated with solving singular integral equations are mitigated by employing innovative analytical and numerical techniques.

There is extensive research in the literature on the application of integral equation techniques to acoustic scattering problems, much of which is discussed in reference [10]. Of particular relevance to the BIEM is the work in references [11-14]. The theory and integral equation terminology presented in reference [11] provide the foundation for the BIEM theoretical discussions. Martinez in references [12-14] has applied singular integral equation methods to the study of sound scattered by a thin, finite-length duct. We expand upon this original research by applying advanced integral equation solution methods and include the ability to treat arbitrary circumferential modes, uniform inflow, enhanced liner models, and a wider range of operating parameters.

\subsection{HELMHOLTZ POTENTIAL REPRESENTATION}

The analysis begins with an analytical expression for the Green's function, $G\left(r, r^{\prime}, Z-Z^{\prime}\right)$, for the two-dimensional Helmholtz operator (18a). $G$ satisfies the radiation condition (18h) and can 
be written

$$
G\left(r, r^{\prime}, Z-Z^{\prime}\right)=\frac{1}{2 \pi} \int_{0}^{\pi} \cos m \psi \frac{e^{-i \kappa R}}{R} d \psi
$$

where

$$
R=\sqrt{r^{2}+r^{\prime 2}-2 r r^{\prime} \cos \psi+\left(Z-Z^{\prime}\right)^{2}}
$$

To facilitate integral equation analysis and numerical computation, we introduce an integral operator notation. For an arbitrary integrable surface function $f$, define single and double layer field operators, $\mathbf{S}$ and $\mathbf{d}$, by the equations

and

$$
\mathbf{S}[f](r, Z)=\int_{a}^{b} f\left(Z^{\prime}\right) s\left(r, Z-Z^{\prime}\right) d Z^{\prime}
$$

$$
\mathbf{d}[f](r, Z)=\int_{a}^{b} f\left(Z^{\prime}\right) d\left(r, Z-Z^{\prime}\right) d Z^{\prime}
$$

where the kernels $s$ and $d$ are given by

$$
s\left(r, Z-Z^{\prime}\right)=G\left(r, 1, Z-Z^{\prime}\right)
$$

and

$$
d\left(r, Z-Z^{\prime}\right)=-\frac{\partial G}{\partial r^{\prime}}\left(r, 1, Z-Z^{\prime}\right)
$$

The integrals in (21-22) are well defined for points $(r, Z)$ not on the stretched duct surface and are equivalent to the classical single and double layer potentials. Care must be exercised when evaluating (21-22) on or near the duct surface.

We define additional field operators by calculating the first radial derivative of $\mathbf{s}$ and $\mathbf{d}$. Denote by $\mathbf{S}_{\mathbf{r}}$ and $\mathbf{d}_{\mathbf{r}}$ the operators

$$
\mathbf{s}_{\mathbf{r}}[f](r, Z)=\frac{\partial}{\partial r}\{\mathbf{s}[f](r, Z)\}=\int_{a}^{b} f\left(Z^{\prime}\right) s_{r}\left(r, Z-Z^{\prime}\right) d Z^{\prime}
$$

and

$$
\mathbf{d}_{\mathbf{r}}[f](r, Z)=\frac{\partial}{\partial r}\{\mathbf{d}[f](r, Z)\}=\int_{a}^{b} f\left(Z^{\prime}\right) d_{r}\left(r, Z-Z^{\prime}\right) d Z^{\prime},
$$

where 


$$
s_{r}\left(r, Z-Z^{\prime}\right)=\frac{\partial s}{\partial r}\left(r, Z-Z^{\prime}\right)
$$

and

$$
d_{r}\left(r, Z-Z^{\prime}\right)=\frac{\partial d}{\partial r}\left(r, Z-Z^{\prime}\right)
$$

By applying the Helmholtz potential representation, the scattered pressure can be written as a sum of single and double layer Helmholtz potentials yielding

$$
Q_{s}(r, Z)=\mathbf{s}\left[q_{1}\right](r, Z)+\mathbf{d}\left[q_{2}\right](r, Z)
$$

Equation (29) is a solution of (18a) and (18h). The unknown layer densities $q_{1}$ and $q_{2}$ are related to the jumps in acoustic pressure and its radial derivative across the duct surface. The densities are a solution to a system of hypersingular integral equations, which we derive below, and once determined provide input to (29) for the pointwise calculation of the acoustic pressure field.

\subsection{SURFACE OPERATOR NOTATION}

To apply the boundary conditions (18c-e) to the potential representation (29), it is necessary to evaluate directly the single and double layer potentials and their derivatives on the stretched duct surface. The resulting integrals yield one-dimensional surface operators with singular kernels that require analytical treatment for their evaluation.

For $Z \in[a, b]$ and sufficiently smooth $f$, we define the surface operators $\mathbf{S}$ and $\mathbf{D}$ by directly evaluating the field operators (21-22) on the duct surface. Thus

$$
\mathbf{S}[f](Z)=\int_{a}^{b} f\left(Z^{\prime}\right) S\left(Z-Z^{\prime}\right) d Z^{\prime}
$$

and

$$
\mathbf{D}[f](Z)=\int_{a}^{b} f\left(Z^{\prime}\right) D\left(Z-Z^{\prime}\right) d Z^{\prime}
$$

where the kernels $S$ and $D$ are given by

$$
S\left(Z-Z^{\prime}\right)=s\left(1, Z-Z^{\prime}\right)
$$


and

$$
D\left(Z-Z^{\prime}\right)=d\left(1, Z-Z^{\prime}\right)
$$

Similarly, we form the operators $\mathbf{S}_{\mathbf{r}}$ and $\mathbf{D}_{\mathbf{r}}$ by direct evaluation of the differentiated field operators (25-26) which yields

$$
\mathbf{S}_{\mathbf{r}}[f](Z)=\int_{a}^{b} f\left(Z^{\prime}\right) S_{r}\left(Z-Z^{\prime}\right) d Z^{\prime}
$$

and

$$
\mathbf{D}_{\mathbf{r}}[f](Z)=\int_{a}^{b} f\left(Z^{\prime}\right) D_{r}\left(Z-Z^{\prime}\right) d Z^{\prime}
$$

where

$$
S_{r}\left(Z-Z^{\prime}\right)=\frac{\partial s}{\partial r}\left(1, Z-Z^{\prime}\right)
$$

and

$$
D_{r}\left(Z-Z^{\prime}\right)=\frac{\partial d}{\partial r}\left(1, Z-Z^{\prime}\right)
$$

\subsection{SURFACE KERNEL PROPERTIES}

The kernels (32-33) and (36-37) are singular for $Z-Z^{\prime}=0$. It is important both theoretically and computationally to determine the precise nature of the singularities. We analytically separate the singular portions of the kernels from the bounded parts by applying small argument analysis. Details are given in appendix A. In equations (38-41) below, the kernels are written as a sum of singular and bounded terms. The bounded parts of the kernels are denoted by a superscript $B$ and are written out explicitly in appendix A.

$$
\begin{gathered}
S\left(Z-Z^{\prime}\right)=-\frac{1}{2 \pi} \ln \left|Z-Z^{\prime}\right|+S^{B}\left(Z-Z^{\prime}\right) \\
D\left(Z-Z^{\prime}\right)=-\frac{1}{4 \pi} \ln \left|Z-Z^{\prime}\right|+D^{B}\left(Z-Z^{\prime}\right) \\
S_{r}\left(Z-Z^{\prime}\right)=-D\left(Z-Z^{\prime}\right)
\end{gathered}
$$




$$
D_{r}\left(Z-Z^{\prime}\right)=-\frac{1}{2 \pi\left(Z-Z^{\prime}\right)^{2}}+\frac{4\left(\kappa^{2}-m^{2}\right)+3}{16 \pi} \ln \left|Z-Z^{\prime}\right|+D_{r}^{B}\left(Z-Z^{\prime}\right)
$$

The leading behavior of the kernels (38-40) is logarithmic. Therefore, the associated operators are weakly singular. The leading term of the kernel (41) is of the strongly singular Hadamard type [15]. Consequently, the integral in (35) is divergent and must be interpreted in the Hadamard finite part sense.

\subsection{LAYER CONTINUITY PROPERTIES}

Using the above surface operator notation, we state well-known continuity properties for the single and double layer field operators (21-22) and their radial derivatives (25-26) as field points approach the stretched duct surface from the interior or exterior of the duct. For a sufficiently smooth surface function $f$, we have the following results [8]:

$$
\begin{gathered}
\lim _{r \rightarrow 1^{ \pm}} \mathbf{s}[f](r, Z)=\mathbf{S}[f](Z) \\
\lim _{r \rightarrow 1^{ \pm}} \mathbf{d}[f](r, Z)=\mp \frac{1}{2} f(Z)+\mathbf{D}[f](Z) \\
\lim _{r \rightarrow 1^{ \pm}} \mathbf{S}_{\mathbf{r}}[f](r, Z)=\mp \frac{1}{2} f(Z)-\mathbf{D}[f](Z) \\
\lim _{r \rightarrow 1^{ \pm}} \mathbf{d}_{\mathbf{r}}[f](r, Z)=\mathbf{D}_{\mathbf{r}}[f](Z)
\end{gathered}
$$

Applying (42-45) to (29) establishes the formulas

$$
Q_{s}^{+}(Z)-Q_{s}^{-}(Z)=-q_{2}(Z)
$$

and

$$
\left(\frac{\partial Q_{s}}{\partial r}\right)^{+}(Z)-\left(\frac{\partial Q_{s}}{\partial r}\right)^{-}(Z)=-q_{1}(Z)
$$

which relate the layer densities to the jumps in scattered pressure and its radial derivative across the stretched duct surface. 


\subsection{BOUNDARY INTEGRAL EQUATIONS}

By applying the continuity results (42-47), we replace the boundary conditions (18c-e) with an equivalent set of integral equations for the unknown surface functions $q_{1}$ and $q_{2}$ in terms of the known incident pressure and its radial derivative.

The exterior boundary condition (18c) can be written

$$
-\left(\frac{1}{2} \mathbf{I}+\mathbf{D}\right)\left[q_{1}\right](Z)+\mathbf{D}_{\mathbf{r}}\left[q_{2}\right](Z)=-\frac{\partial Q_{i}}{\partial r}(1, Z) \quad Z \in(a, b),
$$

where $\mathbf{I}$ is the identity operator. This integral equation is hypersingular due to the presence of the operator $\mathbf{D}_{\mathbf{r}}$. It is worth mentioning here that in many applications, such as lifting wing problems, it is customary to work with (48) after performing an axial integration (recall the comments following equations (14-15)). In this case, the dominant kernel has a less singular Cauchy term. However, no advantage is obtained by this extra calculation because the singular operators are computed analytically. Furthermore, for numerical work, we find that working with the integrated version of (48) is much more cumbersome.

The interior boundary condition (18d) is replaced by the sum of (18c) and (18d) yielding the system of equations

$$
-e^{-i \frac{\kappa}{M} Z} q_{1}(Z)+\frac{i M^{2} \alpha}{\beta^{3} \kappa} \frac{d^{2}}{d Z^{2}}\left(e^{-i \frac{\kappa}{M} Z} Q^{-}\right)(Z)=0 \quad Z \in(a, b)
$$

and

$$
Q^{-}(Z)=Q_{i}(1, Z)+\mathbf{S}\left[q_{1}\right](Z)+\left(\frac{1}{2} \mathbf{I}+\mathbf{D}\right)\left[q_{2}\right](Z) \quad Z \in(a, b)
$$

Equations (49-50) can be combined to yield a single equation by computing two tangential derivatives of $Q^{-}$. The resulting integral equation contains both Cauchy and Hadamard type kernels. It is computationally simpler to introduce a third unknown function, namely $Q^{-}$, and 
work with the integro-differential equations (49-50). We expound on this in the integral equation solution section 4.4. For small Mach number and large $\kappa$, (49-50) can be approximated by the much simpler equation

$$
\left(\beta^{3} \mathbf{I}+i \alpha \kappa \mathbf{S}\right)\left[q_{1}\right](Z)+i \alpha \kappa\left(\frac{1}{2} \mathbf{I}+\mathbf{D}\right)\left[q_{2}\right](Z)=-i \alpha \kappa Q_{i}(1, Z) \quad Z \in(a, b)
$$

The boundary integral equations are supplemented by (18e), which after substitution of the radial derivative of (29) produces the auxiliary equation

$$
\int_{-\infty}^{a} e^{-i \frac{\kappa}{M} Z^{\prime}}\left\{\frac{\partial Q_{i}}{\partial r}\left(1, Z^{\prime}\right)+\mathbf{S}_{\mathbf{r}}\left[q_{1}\right]\left(1, Z^{\prime}\right)+\mathbf{d}_{\mathbf{r}}\left[q_{2}\right]\left(1, Z^{\prime}\right)\right\} d Z^{\prime}=0
$$

As mentioned earlier, this equation is satisfied trivially for $M=0$ and provides no additional information.

If the interior wall is hard $(\alpha \equiv 0)$, then equations (48-52) reduce to the familiar Neumann problem

$$
\begin{gathered}
q_{1}(Z)=0 \quad Z \in(a, b), \\
\mathbf{D}_{\mathbf{r}}\left[q_{2}\right](Z)=-\frac{\partial Q_{i}}{\partial r}(1, Z) \quad Z \in(a, b),
\end{gathered}
$$

with auxiliary condition

$$
\int_{-\infty}^{a} e^{-i \frac{\kappa}{M} Z^{\prime}}\left\{\frac{\partial Q_{i}}{\partial r}\left(1, Z^{\prime}\right)+\mathbf{d}_{\mathbf{r}}\left[q_{2}\right]\left(1, Z^{\prime}\right)\right\} d Z^{\prime}=0
$$

The solution of (53-55) was considered in reference [1].

\subsection{EDGE CONDITIONS}

The system of integral equations (48-52) admits infinitely many solutions and must be supplemented with additional conditions for uniqueness. The as yet unsatisfied BVP edge 
conditions (18f-g) provide the required constraints by restricting the behavior of the double layer density, $q_{2}$, at the duct leading and trailing edges. We omit the edge condition derivations, which involves asymptotic expansions of the hypersingular portion of the singular integral equation (48). See reference [16] for a detailed discussion of this analysis.

We finalize the boundary integral equation formulation by stating the asymptotic behavior of $q_{2}$ near the duct edges:

$$
q_{2}(Z)=O(\sqrt{Z-a}) \quad Z \rightarrow a^{+}
$$

and

$$
q_{2}(Z)=O\left(\frac{M}{\sqrt{b-Z}}\right)+O(\sqrt{b-Z}) \quad Z \rightarrow b^{-}
$$

Note that for $M>0, q_{2}$ has an infinite singularity at the duct leading edge, which is a well-known result in lifting surface theory. This term disappears for the no inflow case.

\subsection{BOUNDARY INTEGRAL EQUATION SUMMARY}

We have succeeded in replacing the BVP (18a-h) by an equivalent boundary integral equation formulation which we now summarize.

For $0 \leq M<1$, the $m$-th circumferential mode of total acoustic pressure is given by

$$
Q(r, Z)=\left\{\begin{array}{l}
Q_{i}(r, Z)+\mathbf{S}\left[q_{1}\right](r, Z)+\mathbf{d}\left[q_{2}\right](r, Z) \quad(r, Z) \text { not on stretched duct surface } \\
Q_{i}(r, Z)+\mathbf{S}\left[q_{1}\right](Z)+\left(\mp \frac{1}{2} \mathbf{I}+\mathbf{D}\right)\left[q_{2}\right](Z) \quad Z \in(a, b) \quad r \rightarrow \pm 1
\end{array},\right.
$$

where $q_{1}$ and $q_{2}$ satisfy

1) the system of strongly singular integral equations

$$
-\left(\frac{1}{2} \mathbf{I}+\mathbf{D}\right)\left[q_{1}\right](Z)+\mathbf{D}_{\mathbf{r}}\left[q_{2}\right](Z)=-\frac{\partial Q_{i}}{\partial r}(1, Z) \quad Z \in(a, b),
$$




$$
\begin{aligned}
& -e^{-i \frac{\kappa}{M} Z} q_{1}(Z)+\frac{i M^{2} \alpha}{\beta^{3} \kappa} \frac{d^{2}}{d Z^{2}}\left(e^{-i \frac{\kappa}{M} Z} Q^{-}\right)(Z)=0 \quad Z \in(a, b) \\
& Q^{-}(Z)=Q_{i}(1, Z)+\mathbf{S}\left[q_{1}\right](Z)+\left(\frac{1}{2} \mathbf{I}+\mathbf{D}\right)\left[q_{2}\right](Z) \quad Z \in(a, b),
\end{aligned}
$$

2) the auxiliary condition

$$
\int_{-\infty}^{a} e^{-i \frac{\kappa}{M} Z^{\prime}}\left\{\frac{\partial Q_{i}}{\partial r}\left(1, Z^{\prime}\right)+\mathbf{S}_{\mathbf{r}}\left[q_{1}\right]\left(1, Z^{\prime}\right)+\mathbf{d}_{\mathbf{r}}\left[q_{2}\right]\left(1, Z^{\prime}\right)\right\} d Z^{\prime}=0
$$

and 3) the edge conditions

$$
\begin{gathered}
q_{2}(Z)=O(\sqrt{Z-a}) \quad Z \rightarrow a^{+} \\
q_{2}(Z)=O\left(\frac{M}{\sqrt{b-Z}}\right)+O(\sqrt{b-Z}) \quad Z \rightarrow b^{-} .
\end{gathered}
$$

For coannular ducts, additional integral operators and layer densities must be defined to accommodate both ducts. In this case, (57) and (58a-f) have to be coupled to similar sets of equations to account for scattering by the second duct. Theoretically, this procedure does not change the singular character of the integral equation system nor the solution method. However, the computational complexity is nearly double.

\section{§ 4. INTEGRAL EQUATION SOLUTION}

In this section we present details of the numerical solution of (58a-f). Existence and uniqueness results are briefly discussed for the hard wall interior case. A theoretical discussion on the solvability of the entire system (58a-f) is lengthy and will be the subject of a future paper by the authors.

Due to edge conditions for the double layer density (58e-f) we are motivated to seek a solution to (58a-f) in the form 


$$
q_{2}(Z)=A \sqrt{\frac{Z-a}{b-Z}}+\sqrt{(b-Z)(Z-a)} q(Z) \quad Z \in(a, b) .
$$

In (59), $A$ is an unknown constant and $q$ an unknown Hölder continuous function.

There are no restrictions on the edge behavior of the single layer density. We note however, that since the interior wall admittance can be discontinuous it follows from (58b) that $q_{1}$ is also discontinuous at the points of discontinuity in $\alpha$.

Our solution methodology is best understood by first examining the simple case of a hardwall interior with no inflow. We next discuss the hardwall interior case in the presence of inflow, which complicates the solution process due to the infinite singularity in the double layer density at the duct leading edge. Finally, we expand the method to include the liner case.

\subsection{HARDWALL INTERIOR WITH NO INFLOW}

We simplify the analysis by the introduction of additional operator notation. Let $f$ be an arbitrary Hölder continuous function and let $\mathbf{K}$ denote any of the previously defined integral operators, then we define new operators $\mathbf{K}^{ \pm}$by the formulas

$$
\mathbf{K}^{ \pm}[f](Z)=\mathbf{K}\left[\left(b-Z^{\prime}\right)^{ \pm 1 / 2}\left(Z^{\prime}-a\right)^{1 / 2} f\right](Z) \quad Z \in(a, b) .
$$

For $M=0$ and $\alpha \equiv 0$, application of (59-60) to (58a-f) produces the single integral equation

$$
\mathbf{D}_{\mathbf{r}}^{+}[q](Z)=-\frac{\partial Q_{i}}{\partial r}(1, Z) \quad Z \in(a, b)
$$

Equation (61) is a one-dimensional, first kind integral equation with a Hadamard kernel. Integral equations of this type appear frequently in the literature and have been applied to problems in acoustics, aerodynamics, elasticity, and electrodynamics (see references [17-18], for example).

Due to the appearance of the weight function $\sqrt{(b-Z)(Z-a)}$, it is natural to seek a solution 
to $(61)$ in the form

$$
q(Z)=\sum_{n=0}^{\infty} a_{n} U_{n}\left(\frac{2 Z-a-b}{b-a}\right)
$$

It is shown in reference [19] that if the source term in (61) is expandable in a series of second kind Chebyshev polynomials, then (61) is uniquely satisfied by a series solution such as (62) except possibly at a countable set of eigenfrequencies. In section 5.2, we demonstrate numerically that some of the eigenfrequencies of $\mathbf{D}_{\mathbf{r}}^{+}$correspond approximately to the transverse resonant frequencies of the infinite duct problem.

In practice, (62) is truncated and the unknown constants $a_{n}$ must be determined numerically, usually by some projection technique such as the method of collocation or Galerkin. The collocation method is simple to implement and requires fewer calculations than other popular methods. For these reasons it is the solution method of choice here.

To apply the collocation method, we consider the approximate solution

$$
\bar{q}(Z)=\sum_{n=0}^{N_{2}} a_{n} U_{n}\left(\frac{2 Z-a-b}{b-a}\right)
$$

and define $N_{2}+1$ distinct collocation points

$$
\left\{Z_{j}\right\}_{j=1}^{N_{2}+1} \quad Z_{j} \in(a, b) \quad \forall j
$$

We next require the approximate solution to satisfy (61) at the collocation points, which yields the linear system of equations

$$
\sum_{n=0}^{N_{2}} a_{n} \mathbf{D}_{\mathbf{r}}^{+}\left[U_{n}\right]\left(Z_{j}\right)=-\frac{\partial Q_{i}}{\partial r}\left(1, Z_{j}\right) \quad j=1, \ldots, N_{2}+1
$$

for the $a_{n}$ 's. Golberg has shown that if the collocation points are chosen as the zeroes of $U_{N_{2}+1}$, then (65) is solvable, except at eigenfrequencies, and the series (63) converges to the actual 
solution $q[19]$.

The evaluation of (65) involves the calculation of integrals with finite-part and logarithmic integrands. To avoid time consuming numerical integration of these terms, we apply the analytical results of appendixes A and B. The bounded portion of the integral operator is computed using Gaussian quadrature with weights and nodes based on the second kind Chebyshev polynomials. The above combination of collocation points and numerical integration scheme yields results as accurate as those obtained by the more computationally intensive Galerkin method [19].

\subsection{HARDWALL INTERIOR WITH INFLOW}

For $\alpha \equiv 0$ and $0<M<1$ equations (58a-f) reduce to

$$
A \mathbf{D}_{\mathbf{r}}^{-}[1](Z)+\mathbf{D}_{\mathbf{r}}^{+}[q](Z)=-\frac{\partial Q_{i}}{\partial r}(1, Z) \quad Z \in(a, b)
$$

and

$$
\int_{-\infty}^{a} e^{-i \frac{\kappa}{M} Z^{\prime}}\left\{\frac{\partial Q_{i}}{\partial r}\left(1, Z^{\prime}\right)+A \mathbf{d}_{\mathbf{r}}^{-}[1]\left(1, Z^{\prime}\right)+\mathbf{d}_{\mathbf{r}}^{+}[q]\left(1, Z^{\prime}\right)\right\} d Z^{\prime}=0
$$

In (66-67), $A$ and $q(Z)$ are unknown. We briefly show, under certain conditions, that if the no inflow problem (61) is uniquely solvable, then so is (66-67). Furthermore, under the same conditions $A$ and $q(Z)$ can be determined separately which we also prove below.

Define the linear functionals $\mathbf{g}_{\mathbf{d}}^{ \pm}$and the known constant $C_{0}$ by the relations

$$
\mathbf{g}_{\mathbf{d}}^{ \pm}[f]=\int_{-\infty}^{a} e^{-i \frac{\kappa}{M} Z^{\prime}} \mathbf{d}_{\mathbf{r}}^{ \pm}[f]\left(1, Z^{\prime}\right) d Z^{\prime}
$$

and

$$
C_{0}=-\int_{-\infty}^{a} e^{-i \frac{\kappa}{M} Z^{\prime}} \frac{\partial Q_{i}}{\partial r}\left(1, Z^{\prime}\right) d Z^{\prime}
$$

Using (68-69) we rewrite (67) as 


$$
A \mathbf{g}_{\mathrm{d}}^{-}[1]+\mathbf{g}_{\mathrm{d}}^{+}[q]=C_{0} .
$$

Assume that conditions are such that (61) is uniquely solvable, then the operator $\mathbf{D}_{\mathbf{r}}^{+}$has a left inverse, which we denote by $\left(\mathbf{D}_{\mathbf{r}}^{+}\right)^{-1}$. Consequently, we "solve" (66) for $q$ to yield

where

$$
q(Z)=\phi_{1}(Z)+A \phi_{2}(Z) \quad Z \in(a, b)
$$

$$
\begin{gathered}
\phi_{l}(Z)=-\left(\mathbf{D}_{\mathbf{r}}^{+}\right)^{-l}\left[\frac{\partial Q_{i}}{\partial r}\right](Z) \quad Z \in(a, b), \\
\phi_{2}(Z)=-\mathbf{D}_{\mathbf{1}}[1](Z) \quad Z \in(a, b),
\end{gathered}
$$

and

$$
\mathbf{D}_{\mathbf{1}}=\left(\mathbf{D}_{\mathbf{r}}^{+}\right)^{-1} \mathbf{D}_{\mathbf{r}}^{-}
$$

Note that $\phi_{1}$ and $\phi_{2}$ are known functions.

We obtain an explicit expression for the unknown constant $A$ in terms of known quantities by applying (71-74) to (70). This substitution gives

$$
A=\frac{C_{0}-\mathbf{g}_{\mathbf{d}}^{+}\left[\phi_{1}\right]}{\mathbf{g}_{\mathbf{d}}^{-}[1]-\mathbf{g}_{\mathbf{d}}^{+}\left[\phi_{2}\right]} .
$$

If $\mathbf{g}_{\mathrm{d}}^{-}[1] \neq \mathbf{g}_{\mathrm{d}}^{+}\left[\phi_{2}\right]$, it follows that $A$ is finite and (71) defines a meaningful expression for $q$.

The above results suggest the following algorithm for solving the hardwall interior integral equations with inflow:

Step 1) Solve the no inflow case, i.e., invert $\mathbf{D}_{\mathbf{r}}^{+}$.

Step 2) Calculate $A$ via (72-75).

Step 3) Calculate $q$ using (71) and (75).

Theoretically, this approach is equivalent to the no inflow case, which implies that the results of Golberg apply [19]. Furthermore, since the computational time required for the calculation of 
the functionals (68) is negligible compared to the time needed to compute the matrix for $\mathbf{D}_{\mathbf{r}}^{+}$, it follows that the above algorithm is also computationally equivalent to the no inflow case.

In reference [1], a slightly different approach was used to separate $A$ and $q$. The resulting algorithms were subsequently shown to be convergent [20].

\subsection{LINED INTERIOR WITH NO INFLOW}

In this case, (58a-f) reduce to the system of integral equations

$$
\begin{gathered}
(\mathbf{I}+i \alpha \kappa \mathbf{S})\left[q_{1}\right](Z)+i \alpha \kappa\left(\frac{1}{2} \mathbf{I}^{+}+\mathbf{D}^{+}\right)[q](Z)=-i \alpha \kappa Q_{i}(1, Z) \quad Z \in(a, b) . \\
-\left(\frac{1}{2} \mathbf{I}+\mathbf{D}\right)\left[q_{1}\right](Z)+\mathbf{D}_{\mathbf{r}}^{+}[q](Z)=-\frac{\partial Q_{i}}{\partial r}(1, Z) \quad Z \in(a, b)
\end{gathered}
$$

We defer a detailed theoretical discussion of the singular system (76a-b) to a future publication and, instead, focus on the solution technique.

To solve (76a-b) numerically we again apply the collocation method. The double layer density $q$ is approximated as in the no inflow, hardwall case. Since there are no restrictions on the single layer edge behavior and the weight function for the Legendre polynomials is unity we seek an approximation to $q_{1}$ of the form

$$
\bar{q}_{l}(Z)=\sum_{n=0}^{N_{l}} b_{n} P_{n}\left(\frac{2 Z-a-b}{b-a}\right)
$$

Further motivation is provided by the fact that the numerical accuracy achievable by the expansion (77) is commensurate with that for the double layer density approximation (63).

We collocate (76a) at the zeroes of $P_{N_{1}+1},\left\{Y_{j}\right\}_{j=1}^{N_{2}+1}$, and (76b) at the zeroes of $U_{N_{2}+1}$, $\left\{Z_{j}\right\}_{j=1}^{N_{2}+1}$, yielding the $\left(N_{1} N_{2}\right) \times\left(N_{1} N_{2}\right)$ linear system 


$$
\begin{gathered}
\sum_{n=0}^{N_{l}} b_{n}(\mathbf{I}+i \alpha \kappa \mathbf{S})\left[P_{n}\right]\left(Y_{j}\right)+i \alpha \kappa \sum_{n=0}^{N_{l}} a_{n}\left(\frac{1}{2} \mathbf{I}^{+}+\mathbf{D}^{+}\right)\left[U_{n}\right]\left(Y_{j}\right) \\
=-i \alpha \kappa Q_{i}\left(1, Y_{j}\right) \quad j=1, \ldots, N_{1} \\
-\sum_{n=0}^{N_{l}} b_{n}\left(\frac{1}{2} \mathbf{I}+\mathbf{D}\right)\left[P_{n}\right]\left(Z_{j}\right)+\sum_{n=0}^{N_{l}} a_{n} \mathbf{D}_{\mathbf{r}}^{+}\left[U_{n}\right]\left(Z_{j}\right)=-\frac{\partial Q_{i}}{\partial r}\left(1, Z_{j}\right) \quad j=1, \ldots, N_{2}
\end{gathered}
$$

for the unknown expansion coefficients. No theoretical results exist regarding the solvability and convergence of this numerical method. However, computational evidence suggests that the linear system (78a-b) is stable and the discretized solutions converge to the actual solution of the system of integral equations (76a-b).

\subsection{LINED INTERIOR WITH INFLOW}

Define the functional $\mathbf{g}_{\mathbf{s}}$ by the formula

$$
\mathbf{g}_{\mathbf{s}}[f]=\int_{-\infty}^{a} e^{-i \frac{\kappa}{M} Z^{\prime}} \mathbf{S}_{\mathbf{r}}[f]\left(1, Z^{\prime}\right) d Z^{\prime} .
$$

For a lined interior and $0<M<1$, equations (58a-f) assume their most complex form:

$$
\begin{gathered}
-e^{-i \frac{\kappa}{M} Z} q_{1}(Z)+\frac{i M^{2} \alpha}{\beta^{3} \kappa} \frac{d^{2}}{d Z^{2}}\left(e^{-i \frac{\kappa}{M} Z} Q^{-}\right)(Z)=0 \quad Z \in(a, b) \\
Q^{-}(Z)=Q_{i}(1, Z)+\mathbf{S}\left[q_{1}\right](Z)+ \\
A\left(\frac{1}{2} \mathbf{I}^{-}+\mathbf{D}^{-}\right)[l](Z)+\left(\frac{1}{2} \mathbf{I}^{+}+\mathbf{D}^{+}\right)[q](Z) \quad Z \in(a, b) \\
-\left(\frac{1}{2} \mathbf{I}+\mathbf{D}\right)\left[q_{1}\right](Z)+\mathbf{D}_{\mathbf{r}}^{+}[q](Z)+A \mathbf{D}_{\mathbf{r}}^{-}[1](Z)=-\frac{\partial Q_{i}}{\partial r}(1, Z) \quad Z \in(a, b) \\
\mathbf{g}_{\mathbf{s}}\left[q_{1}\right]+A \mathbf{g}_{\mathbf{d}}^{-}[1]+\mathbf{g}_{\mathbf{d}}^{+}[q]=C_{0}
\end{gathered}
$$

Equations (80a-b) can be combined to form one equation by substituting (80a) into (80b). The resulting hypersingular integral equation contains two axial derivatives of the single and 
double layer surface operators. It is far simpler computationally to introduce a third unknown function $Q^{-}$, which we again approximate by a finite sum of Legendre polynomials. A linear system is obtained by applying the usual collocation method and the unknown constant $A$ is determined by a manner similar to that used for the hardwall case.

We simplify the solution process even further by replacing (58b-c) with the approximate equation (51). Thus, (80a-b) reduce to

$$
\begin{aligned}
& \left(\beta^{3} \mathbf{I}+i \alpha \kappa \mathbf{S}\right)\left[q_{1}\right](Z)+i \alpha \kappa A\left(\frac{1}{2} \mathbf{I}^{-}+\mathbf{D}^{-}\right)[1](Z)+ \\
& i \alpha \kappa\left(\frac{1}{2} \mathbf{I}^{+}+\mathbf{D}^{+}\right)[q](Z)=-i \alpha \kappa Q_{i}(1, Z) \quad Z \in(a, b)
\end{aligned} .
$$

This equation is valid for small values of the parameter $M / \kappa$ and is similar to the no inflow integral equation (76a). The system of equations (81) and (80c-d) is then solved by first separating the determination of the constant $A$ and then applying the collocation scheme described in the previous section.

\section{$\S 5$. RESULTS}

In this section, computational results are presented that demonstrate BIEM features. We first discuss the ducted fan noise prediction code TBIEM3D [6] and its application to parametric noise reduction studies. Next, the relationship between the finite duct BIEM and infinite duct prediction methods is established by analyzing the spectral content of the BIEM interior pressure field. Lastly, we illustrate BIEM liner capability by conducting a numerical experiment in which the effects of interior admittance distributions on radiated sound are studied. 


\subsection{THE DUCTED FAN NOISE PREDICTION CODE TBIEM3D}

A PC based computer program, TBIEM3D (Thin duct, BIEM, Three-Dimensional), has been developed which incorporates the BIEM solution features [6]. Results from a typical TBIEM3D calculation are shown in figure 2 . In this simulation, 20 spinning point axial dipoles, situated on a disk in the middle of a short duct, generate the twentieth circumferential mode of acoustic pressure and its harmonics. The duct walls are hard and sound propagates in the presence of a uniform axial flow with $M=0.4$. This configuration was chosen to approximate the thrust component of loading noise produced by a 20 -bladed fan. Figure 2 a shows a cut-a-way view of the engine duct in which contours of instantaneous acoustic pressure for the $m=20$ circumferential mode are plotted in the duct interior. Conditions are such that only one radial mode is cut-on. Doppler effects and reflections from the duct openings are included. Portions of sound reaching the duct ends radiate to the farfield as indicated in figure $2 \mathrm{~b}$. The combined acoustic field in figure 2 is composed of 10,000 field calculations and consumed approximately 45 minutes computational time on a laptop personal computer with a Pentium 133 processor and 32 megabytes of random access memory.

To demonstrate the effectiveness of TBIEM3D as a noise prediction tool, the authors and coworkers have applied the code in several engineering studies. In reference [21], TBIEM3D was used to quantify the concept of generalized radiation impedance and to assess the accuracy of combining infinite duct propagation results with Rayleigh's radiation formula. Results from this research clearly illustrate the similarities and differences between the BIEM and infinite duct prediction methods.

TBIEM3D has been applied to both passive and active noise control research [22-25]. For given liner length and location, optimal liner impedances were calculated for a host of operating 
situations in which circumferential mode number, inflow Mach number, and excitation frequency were varied [22]. Optimal liner impedance is also a function of the designer's definition of noise attenuation. In this regard, TBIEM3D can be easily adapted to accommodate noise metrics that are based on the radiated or internal acoustic pressure fields. For example, in reference [22] liner impedance was chosen so that the maximum sound pressure level along an arc of farfield observers in the direction of the principal radial mode was minimized. Active noise control research based on TBIEM3D has focused on using simple point control sources and a feedforward algorithm to minimize the sound along a particular farfield direction produced by a given source configuration [23].

A two dimensional version of the code has been used to examine the effect of a scarf inlet on farfield noise radiation from a short duct [24-25]. Results from these studies indicate that the scarf inlet is very effective at redirecting high order transverse modes away from noise sensitive regions over a wide range of excitation frequencies. This phenomenon is well known at high frequency [26]. Calculations suggest that the scarf inlet also redirects lower order transverse modes but to a lesser degree. In the absence of flow, the scarf inlet has little affect on aft radiation patterns. However, the amount of distortion in the aft region due to the scarf inlet appears to increase with increasing inflow Mach number. It was further shown that the interior acoustic field may be seriously altered by the presence of a scarf inlet, which provides an additional mechanism for passive noise control.

\subsection{HARDWALL INTERIOR: SPECTRAL ANALYSIS}

In this two part numerical experiment, we analyze the spectral contents of the BIEM hardwall interior field and relate the findings to the usual infinite duct eigenfunction expansion. To 
simulate infinite duct conditions with TBIEM3D, we consider a long duct $\left(L_{D}=6.0\right)$ with the source disk located at one end.

For an infinitely long hardwall duct with unit radius, it is well-known that, due to the phenomenon of transverse resonance, the interior Helmholtz problem is unsolvable at the cut-on wavenumbers $\left\{k_{r}^{m n}\right\}_{n=1}^{\infty}$, where $J_{m}^{\prime}\left(k_{r}^{m n}\right)=0$. It is intuitive to assume that the discretized linear system for the long, finite duct problem should experience some ill-conditioning near the infinite duct eigenwavenumbers.

In figure 3, we plot the $L_{l}$ condition number of the $\mathbf{D}_{\mathbf{r}}^{+}$matrix (see equation 65 ) as a function of excitation wavenumber, $k$, for the circumferential mode numbers $m=4$ and $m=10$. The location of the infinite duct resonant wavenumbers are denoted by dashed lines. As expected, serious ill-conditioning occurs near the cut-on wavenumbers. Also present are lesser spikes of illconditioning. The cause of the finite duct ill-conditioning is probably due to the organ-pipe resonance phenomenon. This subject and the viability of the BIEM solution at these organ-pipe eigenwavenumbers will be addressed in a future paper by the authors.

Part two of this experiment involves comparing BIEM interior axial wavenumber spectra with the discrete infinite duct results. The infinite duct axial wavenumbers are given by the formula [3]

$$
k_{z}^{m n}=\frac{\kappa}{\beta}\left[-M \pm \sqrt{1-\left(\frac{k_{r}^{m n}}{\kappa}\right)^{2}}\right]
$$

The duct mode $(m, n)$ is cut-on if $\kappa>k_{r}^{m n}$. To compute the axial spectrum of the BIEM interior field, the acoustic pressure is calculated along a line parallel to the duct axis and analyzed using FFT techniques. Axial BIEM spectra for two separate cases are presented in figure 4 and compared to the infinite duct spectra. Operating conditions were chosen so that three radial 
modes were cut-on. There are two important observations from these results. First, the locations of the BIEM spectral peaks are in excellent agreement with the infinite duct axial wavenumbers. Second, reflections at the duct ends are included and the relative strengths of propagated and reflected modes can be easily determined using BIEM results.

\subsection{SAMPLE LINER CALCULATIONS}

We illustrate BIEM liner capabilities by studying the effect of interior admittance distribution on noise radiation patterns. For this virtual experiment, we consider a short duct, $L_{D}=0.5$, with the source disk located in the middle of the duct. Twenty spinning point axial dipoles generate incident sound in the presence of a uniform inflow with $M=0.4$. The rotational speed of the source disk is chosen such that one radial mode is cut-on.

In figure 5, contours of sound pressure level are plotted in the acoustic field for four different admittance distributions. Figure 5a shows the scattered field for a hardwall interior. Three different liner configurations are considered in figures 5b-d. As shown in figure 5b, a portion of the inlet is lined $(L=0.2)$ with admittance chosen to be optimal in the sense of reference [22]. Significant noise reduction in the forward region is attained. Figure $5 \mathrm{c}$ shows the results of lining a portion of the exhaust duct. For this case, the admittance is not optimal. Some noise reduction in the aft region is apparent with little or no reduction in the forward region. In figure $5 \mathrm{~d}$, both the inlet and exhaust are lined resulting in substantial noise reduction both forward and aft. Each case presented here required several minutes computational time on a Pentium 133 personal computer.

\section{§ 6. CONCLUDING REMARKS}

A boundary integral equation method for the prediction of ducted fan engine noise has been presented. Based on the equations of linearized acoustics, a classical boundary value problem was 
derived in its entirety. The ability to treat uniform, axial inflow and locally reacting, axially segmented liners is included. Using Helmholtz potentials, the BVP was recast in terms of a system of hypersingular boundary integral equations. The extension of the BIEM to coannular ducts was described.

Innovations in the theoretical approach to solving the integral equation system and in the analysis of the integral equation kernels have produced a computationally efficient solution procedure. The ducted fan noise prediction program TBIEM3D incorporates the BIEM solution features and rapidly predicts the sound field produced by the scattering of incident sound by a finite length cylindrical duct.

Unlike infinite duct prediction methods, the BIEM does not require the decomposition of the acoustic field into its radial and axial components. However, spectral evidence presented in the results section clearly establishes the connection between the finite duct BIEM and infinite duct prediction methods. That is, for the hardwall case, the BIEM interior field displays the axial and radial spectral characteristics that are predicted by the infinite duct theory. Furthermore, reflections due to the duct openings are accounted for implicitly without resorting to approximate measures. It was also demonstrated that noise predictions involving lined interiors are easily calculated without the need to solve the complex eigenvalue problem.

It has been shown that TBIEM3D is accurate, executable on inexpensive computer platforms, and applicable over a wide range of operating conditions. Code versatility, ease-of-use, and rapid prediction capability are necessary features for conducting cost-efficient parametric noise reduction analyses. In this regard, the effectiveness of TBIEM3D as an engineering analysis tool has been amply demonstrated both here and in the literature [21-25]. 


\section{§ 7. REFERENCES}

1. M.H. Dunn, J. Tweed, and F. Farassat 1996 AIAA Paper 96-1770. The Prediction of Ducted Fan Engine Noise via a Boundary Integral Equation Method.

2. J.M. Tyler and T.G. Sofrin 1962 SAE Trans.70. Axial Flow Compressor Noise Studies.

3. W. Eversman 1991 Chapter 13 in Aeroacoustics of Flight Vehicles: Theory and Practice. Vol. 2, NASA RP 1258. Theoretical Models for Duct Acoustic Propagation and Radiation.

4. J.F. Groeneweg, T.G. Sofrin, E.J. Rice, and P.R. Gliebe 1991 Chapter 3 in Aeroacoustics of Flight Vehicles: Theory and Practice. Vol. 1, NASA RP 1258. Turbomachinery Noise.

5. R.E. Motsinger and R.E. Kraft 1991 Chapter 14 in Aeroacoustics of Flight Vehicles: Theory and Practice. Vol. 2, NASA RP 1258. Design and Performance of Duct Acoustic Treatment..

6. M.H. Dunn 1997 NASA/CR-97-206232. TBIEM3D - A Computer Program for Predicting Ducted Fan Engine Noise, Version 1.1.

7. F. Farassat and M.H. Dunn 1998 Submitted to Journal of Sound and Vibration .A Simple Derivation of the Acoustic Boundary Condition in the Presence of Flow.

8. V.I. Smirnov 1964 A Course of Higher Mathematics IV. Oxford: Pergamon Press.

9. P.L. Spence 1997 AIAA Paper 97-1651. Ducted Fan Noise Prediction using Wave Envelope Analysis and the Kirchhoff Formula.

10. J.J de Rego Silva 1994 Acoustic and Elastic Wave Scattering using Boundary Elements. Southampton: Computational Mechanics Publications.

11. R.E. Kleinman and G.E. Roach 1974 SIAM Review 16(2). Boundary Integral Equations for the Three-Dimensional Helmholtz Equation.

12. R. Martinez 1987 AIAA Journal 26(4). Diffracting Open-ended Pipe Treated as a Lifting Surface. 
13. R. Martinez 1990 J. Acous. Soc. Am. 87(2). A Boundary Integral Formulation for Thin-walled Shapes of Revolution.

14. R. Martinez 1993 NASA CR 190801. Aeroacoustic Diffraction and Dissipation by a Short Propeller Cowl in Subsonic Flight.

15. J. Hadamard 1923 Lectures on Cauchy's Problems in Partial Differential Equations. New Haven: Yale University Press.

16. A.C. Kaya and F. Erdogan 1987 Q. of App. Math. XLV(3). On the Solution of Integral Equations with Generalized Cauchy Kernel.

17. J. Katz and A. Plotkin 1991 Low Speed Aerodynamics - From Wing Theory to Panel Methods. McGraw-Hill.

18. F. Erdogan 1978 Mechanics Today 4, S. Nemat-Nasser, ed. Mixed Boundary Value Problems.

19. M. Golberg 1983 J. of Integral Equations 5. The Convergence of Several Algorithms for Solving Integral Equations with Finite-Part Kernels.

20. J. Tweed, R. St. John, and M.H. Dunn 1998 Accepted for publication in Applied Math Letters. Algorithms for the Numerical Solution of a Finite-Part Integral Equation.

21. J. Posey, M.H. Dunn, and F. Farassat 1998 AIAA/CEAS Paper 98-2248. Quantification of Inlet Impedance Concept.

22. M.H. Dunn and F. Farassat 1998 AIAA/CEAS Paper 98-2310. Liner Optimization Studies Using the Ducted Fan Noise Prediction Code TBEIM3D.

23. F.V. Hutcheson 1999 Advanced Modeling of Active Control of Fan Noise for Ultra High Bypass Turbofan Engines. Virginia Tech, Blacksburg, Virginia: Ph.D. Dissertation.

24. M.H. Dunn, J. Tweed, and R. St. John 1998 AIAA Paper 98-2202. Sound Radiated from a 
Two-Dimensional Scarf Inlet Using the Noise Prediction Code TBIEM2D.

25. R. St. John 1998 The Solution of Hypersingular Integral Equations with Applications in Acoustics and Fracture Mechanics. Old Dominion University, Norfolk, Virginia: Ph.D. Dissertation.

26. N. Peake 1995 CEAS/AIAA Paper 95-101. A Theoretical Investigation of the Noise Radiation from a Scarfed Intake.

27. F.D. Gakhov 1966 Boundary Value Problems. New York: Dover Publications.

28. Kaya and Erdogan 1987 Q. of App. Math., Vol. XLV(1). On the Solution of Integral Equations with Strongly Singular Kernels. 


\section{$\S 8$. APPENDICES}

\subsection{APPENDIX A: SINGULAR KERNEL ANALYSIS}

We derive the singular surface kernel results (38-41) by applying small argument analysis to the Green's function (19) and its derivatives. Since no simple closed form expression exists for (19), it must be evaluated numerically. Due to the singularities, conventional numerical integration fails when evaluating the Green's function on the stretched duct surface. Thus, analytical methods are required for resolution of the singular terms. For each kernel, the singular portion is separated from the bounded part by subtracting the singular terms from the Green's function integrand. The resulting singular integrals are evaluated in closed form and the remaining integrands are continuous everywhere and present no numerical problems.

From (32) and (19), we have

$$
S\left(Z-Z^{\prime}\right)=\frac{1}{2 \pi} \int_{0}^{\pi} \cos m \psi \frac{e^{-i \kappa R_{l l}}}{R_{l 1}} d \psi
$$

where

$$
R_{l l}=\sqrt{\left(2 \sin \frac{\psi}{2}\right)^{2}+\left(Z-Z^{\prime}\right)^{2}} .
$$

The integrand in (A1) is $O\left(\frac{1}{R_{11}}\right)$ for small $\psi$ and $\left|Z-Z^{\prime}\right|$. Therefore, we seek a function $g_{S}(\psi)$ such that

$$
h_{S}\left(\psi, Z-Z^{\prime}\right) \equiv \cos m \psi e^{-i \kappa R_{I I}}-g_{S}(\psi)=O\left(R_{I I}\right) \quad \psi,\left|Z-Z^{\prime}\right| \rightarrow 0
$$

and the integral

$$
\int_{0}^{\pi} \frac{g_{S}(\psi)}{R_{l 1}} d \psi
$$

is known in terms of elementary functions. One such choice is 
which yields

$$
g_{S}(\psi)=\cos \frac{\psi}{2}
$$

$$
\int_{0}^{\pi} \frac{\cos \frac{\psi}{2}}{R_{11}} d \psi=-\ln \left|Z-Z^{\prime}\right|+\ln \left[2+\sqrt{4+\left(Z-Z^{\prime}\right)^{2}}\right]
$$

Consequently,

$$
S\left(Z-Z^{\prime}\right)=-\frac{1}{2 \pi} \ln \left|Z-Z^{\prime}\right|+S^{B}\left(Z-Z^{\prime}\right)
$$

where

$$
S^{B}\left(Z-Z^{\prime}\right)=\frac{1}{2 \pi} \int_{0}^{\pi} \frac{h_{S}\left(\psi, Z-Z^{\prime}\right)}{R_{11}} d \psi+\frac{1}{2 \pi} \ln (2+W)
$$

and

$$
W=\sqrt{4+\left(Z-Z^{\prime}\right)^{2}}
$$

We apply a similar analysis to the double layer surface kernel to obtain

$$
D\left(Z-Z^{\prime}\right)=-\frac{1}{4 \pi} \ln \left|Z-Z^{\prime}\right|+D^{B}\left(Z-Z^{\prime}\right),
$$

where

$$
D^{B}\left(Z-Z^{\prime}\right)=\frac{1}{4 \pi} \int_{0}^{\pi} \frac{h_{D}\left(\psi, Z-Z^{\prime}\right)}{R_{11}^{3}} d \psi+\frac{1}{4 \pi} \ln (2+W)-\frac{1}{2 \pi W}
$$

and

$$
h_{D}\left(\psi, Z-Z^{\prime}\right)=\left(2 \sin \frac{\psi}{2}\right)^{2}\left[\cos m \psi\left(1+i \kappa R_{11}\right) e^{-i \kappa R_{I I}}-\cos \frac{\psi}{2}\right]
$$

Analysis for the hypersingular kernel (41) proceeds like before producing

$$
D_{r}\left(Z-Z^{\prime}\right)=-\frac{1}{2 \pi\left(Z-Z^{\prime}\right)^{2}}+\frac{4\left(\kappa^{2}-m^{2}\right)+3}{16 \pi} \ln \left|Z-Z^{\prime}\right|+D_{r}^{B}\left(Z-Z^{\prime}\right) .
$$

The expressions for $D_{r}^{B}$ are lengthy and will be given in two parts:

where

$$
D_{r}^{B}\left(Z-Z^{\prime}\right)=D_{1}^{B}\left(Z-Z^{\prime}\right)+D_{2}^{B}\left(Z-Z^{\prime}\right)
$$

$$
D_{l}^{B}\left(Z-Z^{\prime}\right)=-\frac{1}{2 \pi} \int_{0}^{\pi} \frac{h_{l}\left(\psi, Z-Z^{\prime}\right)}{R_{11}^{3}} d \psi-\frac{2 C+\kappa^{2}}{4 \pi} \ln (2+W)+\frac{1+2 C(2+W)}{2 \pi W(2+W)}
$$




$$
\begin{gathered}
D_{2}^{B}\left(Z-Z^{\prime}\right)=-\frac{1}{8 \pi} \int_{0}^{\pi} \frac{h_{2}\left(\psi, Z-Z^{\prime}\right)}{R_{11}^{5}} d \psi-\frac{3}{8 \pi} \ln (2+W)+\frac{4+3 W^{2}}{4 \pi W^{3}}, \\
h_{l}\left(\psi, Z-Z^{\prime}\right)=\cos m \psi \cos \psi\left(1+i \kappa R_{I I}\right) e^{-i \kappa R_{I I}}-\cos \frac{\psi}{2}\left[1+C\left(2 \sin \frac{\psi}{2}\right)^{2}+\frac{\kappa^{2}}{2} R_{I I}^{2}\right], \\
h_{2}\left(\psi, Z-Z^{\prime}\right)=\left(2 \sin \frac{\psi}{2}\right)^{4}\left[\cos m \psi\left(3+3 i \kappa R_{I I}-\kappa^{2} R_{I I}^{2}\right) e^{-i \kappa R_{I I}}-3 \cos \frac{\psi}{2}\right],
\end{gathered}
$$

and

$$
C=-\frac{3+4 m^{2}}{8} .
$$

\subsection{APPENDIX B: SINGULAR INTEGRAL EVALUATIONS}

In solving the system of integral equations (58a-g), hypersingular and logarithmic integrals involving Legendre and second kind Chebyshev polynomials are encountered. We list without proof several pertinent integration results, which can be obtained by applying the PlemeljSokhotski theorem and its logarithmic analog [27]. Some of these results are well-known and appear in the literature (see reference [28], for example).

$$
\begin{aligned}
& \int_{-1}^{1} \frac{\sqrt{1-y^{2}} U_{n}(y)}{(x-y)^{2}} d y=-\pi(n+1) \begin{cases}U_{n}(x) \quad x \in[-1,1] \\
\frac{\left(x+\sqrt{x^{2}-1}\right)^{n+1}}{\sqrt{x^{2}-1}} \quad x<-1\end{cases} \\
& \int_{-1}^{1} \sqrt{1-y^{2}} U_{0}(y) \ln |x-y| d y=\frac{\pi}{4} \begin{cases}T_{2}(x)-2 \ln 2 \quad x \in[-1,1] \\
\left(x+\sqrt{x^{2}-1}\right)^{2}+2 \ln \frac{1}{2}\left(\sqrt{x^{2}-1}-x\right) \quad x<-1\end{cases}
\end{aligned}
$$


$\int_{-1}^{1} \sqrt{1-y^{2}} U_{n}(y) \ln |x-y| d y=\frac{\pi}{2} \begin{cases}\frac{T_{n+2}(x)}{n+2}-\frac{T_{2}(x)}{n} \quad x \in[-1,1] & n>0 \\ \frac{\left(x+\sqrt{x^{2}-1}\right)^{n+2}}{n+2}-\frac{\left(x+\sqrt{x^{2}-1}\right)^{n}}{n} & x<-1\end{cases}$

$\int_{-1}^{1} \sqrt{\frac{1+y}{1-y}} \frac{d y}{(x-y)^{2}}=\pi \begin{cases}0 \quad x \in[-1,1] \\ \frac{1}{(1-x) \sqrt{x^{2}-1}} & x<-1\end{cases}$

$\int_{-1}^{1} \sqrt{\frac{1+y}{1-y}} \ln |x-y| d y=-\pi(x+\ln 2)-\pi \begin{cases}0 & x \in[-1,1] \\ \sqrt{x^{2}-1}-\ln \left(\sqrt{x^{2}-1}-x\right) & x<-1\end{cases}$

$\int_{-1}^{1} P_{0}(y) \ln |x-y| d y=2 Q_{1}(x)+\ln \left|x^{2}-1\right| \quad x \neq \pm 1$

$\int_{-1}^{1} P_{n}(y) \ln |x-y| d y=\frac{2}{2 n+1}\left[Q_{n+1}(x)-Q_{n-1}(x)\right] \quad x \neq \pm 1 \quad n>0$

\section{$\S 9$. NOTATION}

\subsection{PARAMETERS AND VARIABLES}

$\sim \quad$ Denotes that a quantity is dimensional when appearing over a variable

$a \quad$ Axial coordinate of duct trailing edge in stretched, moving frame

$b \quad$ Axial coordinate of duct leading edge in stretched, moving frame

$\tilde{c} \quad$ Ambient sound speed

$J_{m} \quad$ First kind Bessel function of order $m$

$k=\frac{\tilde{r}_{D} \widetilde{\omega}}{\widetilde{c}}$ Characteristic wave number 
$k_{r}^{m n} \quad$ Radial wavenumbers

$k_{z}^{m n} \quad$ Axial wavenumbers

L Liner length

$L_{D} \quad$ Ratio of duct length to duct diameter

$m \quad$ Circumferential mode number

$M \quad=\frac{\widetilde{U}}{\tilde{c}}$ Inflow Mach number

$n \quad$ Radial mode number

$N \quad$ Number of sources

$p^{\prime} \quad$ Eulerian description of total acoustic pressure field

$p_{i}^{\prime} \quad$ Eulerian description of incident acoustic pressure field

$p_{s}^{\prime} \quad$ Eulerian description of scattered acoustic pressure field

$P_{n} \quad$ Legendre polynomials

$Q_{n} \quad$ Associated Legendre functions of the second kind of order zero

$(r, \psi, z) \quad$ Cylindrical coordinates in Eulerian frame

$\tilde{r}_{D} \quad$ Duct radius

$\tilde{t}, t \quad$ Time

$T_{n} \quad$ Chebyshev polynomials of the first kind

$u_{r}^{\prime} \quad$ Eulerian description of the radial component of acoustic velocity

$\widetilde{U} \quad$ Axial speed of duct

$U_{n} \quad$ Chebyshev polynomials of the second kind

Z Axial coordinate in stretched, moving frame 
$\alpha \quad$ Specific acoustic admittance on duct interior surface

$\beta \quad=\sqrt{1-M^{2}}$ Stretching parameter

$\kappa \quad=k / \beta$ Stretched characteristic wave number

$\tilde{\rho}_{0} \quad$ Ambient density

$\tilde{\omega} \quad$ Excitation frequency (radians/second)

$\tilde{\Omega} \quad$ Shaft speed (radians/second)

\subsection{INTEGRAL EQUATION OPERATORS AND KERNELS}

d, $d \quad$ Double layer field operator and kernel

D, $D \quad$ Double layer surface operator and kernel

$D^{B} \quad$ Bounded portion of double layer surface kernel

$\mathbf{d}_{\mathbf{r}}, d_{r} \quad$ Radial derivative of double layer field operator and kernel

$\mathbf{D}_{\mathbf{r}}, D_{r} \quad$ Radial derivative of double layer surface operator and kernel

$D_{r}^{B} \quad$ Bounded portion of double layer radial derivative surface kernel

S, $s \quad$ Single layer field operator and kernel

S, $S \quad$ Single layer surface operator and kernel

$S^{B} \quad$ Bounded portion of single layer surface kernel

$\mathbf{S}_{\mathbf{r}}, s_{r} \quad$ Radial derivative of single layer field operator and kernel

$\mathbf{S}_{\mathbf{r}}, S_{r} \quad$ Radial derivative of single layer surface operator and kernel 


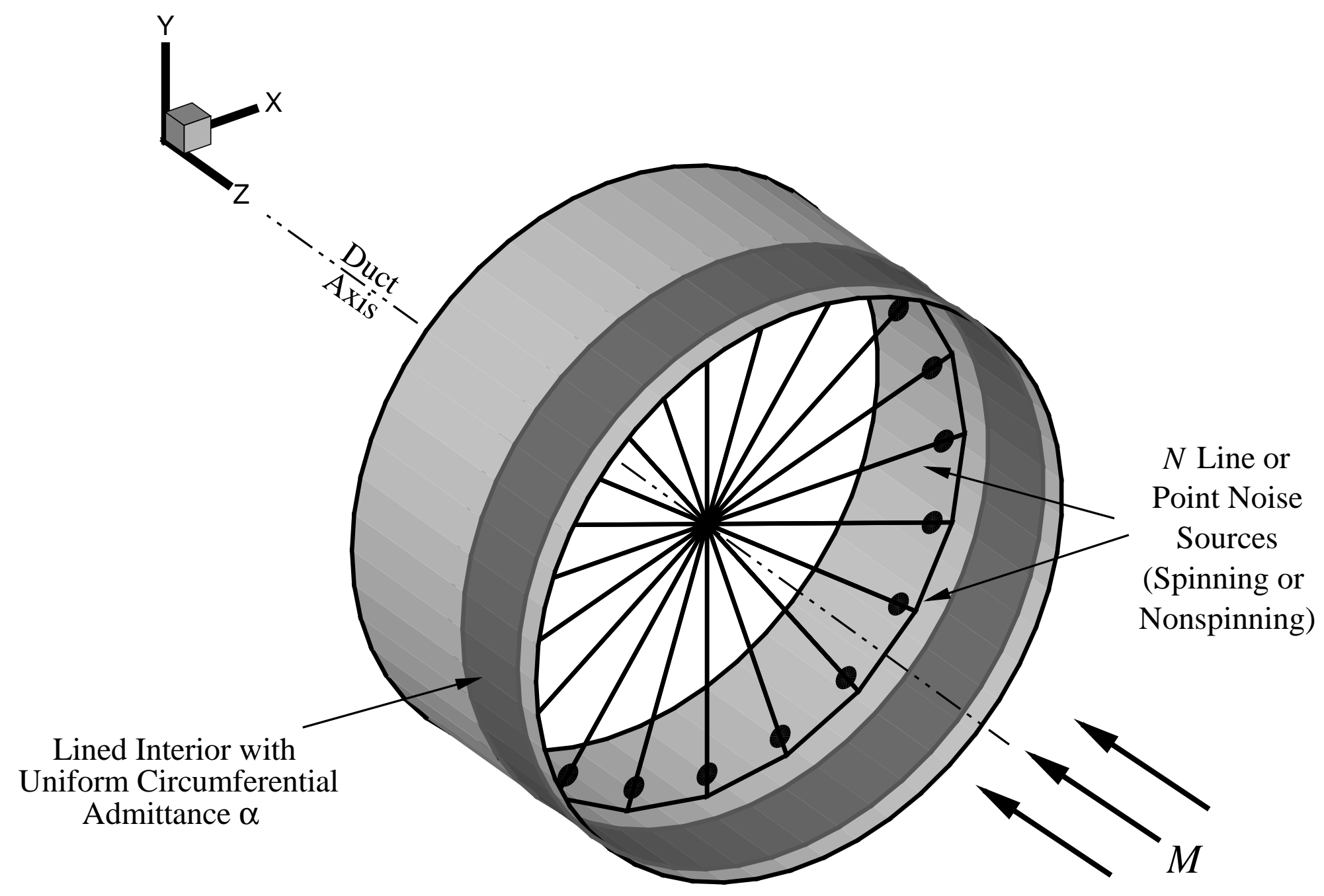

Figure 1: BIEM Geometry - Source Plane Located at $z=0$ 
a)

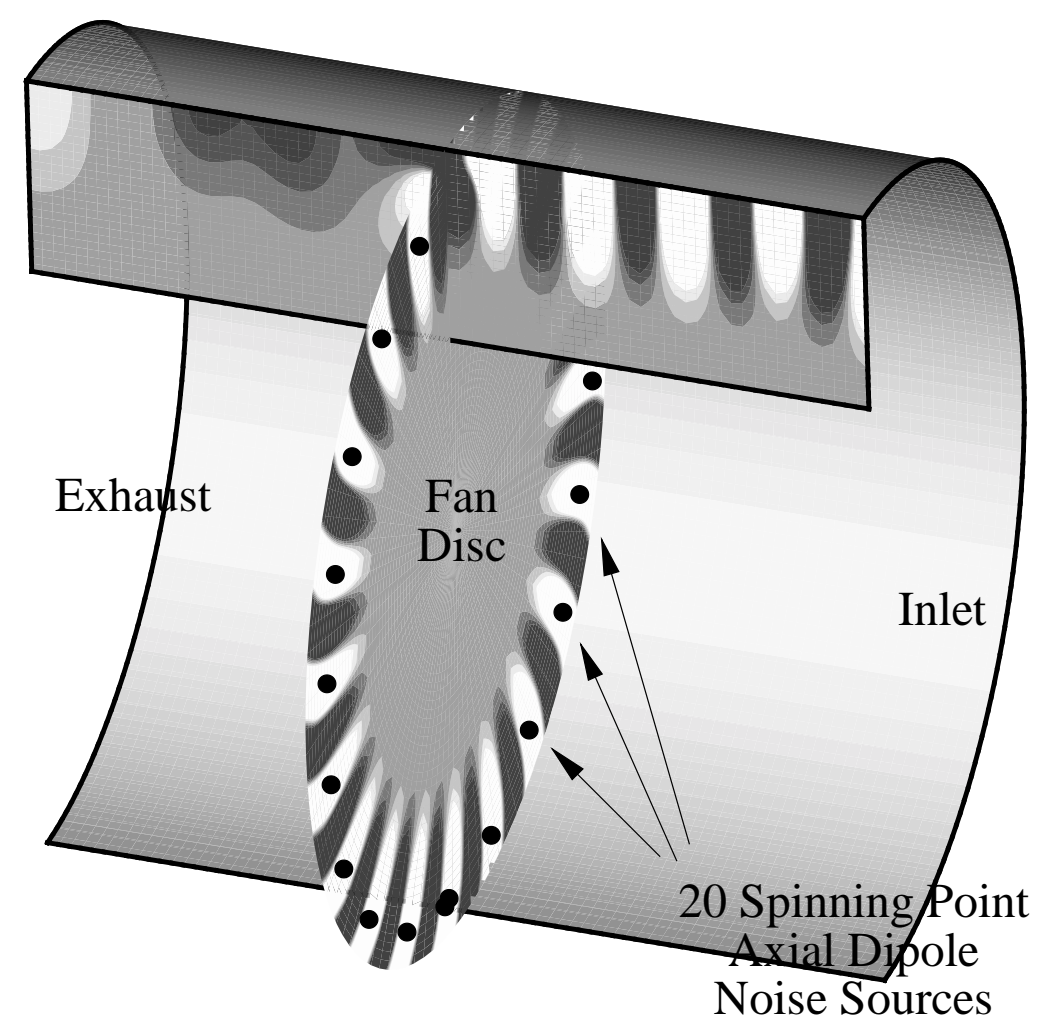

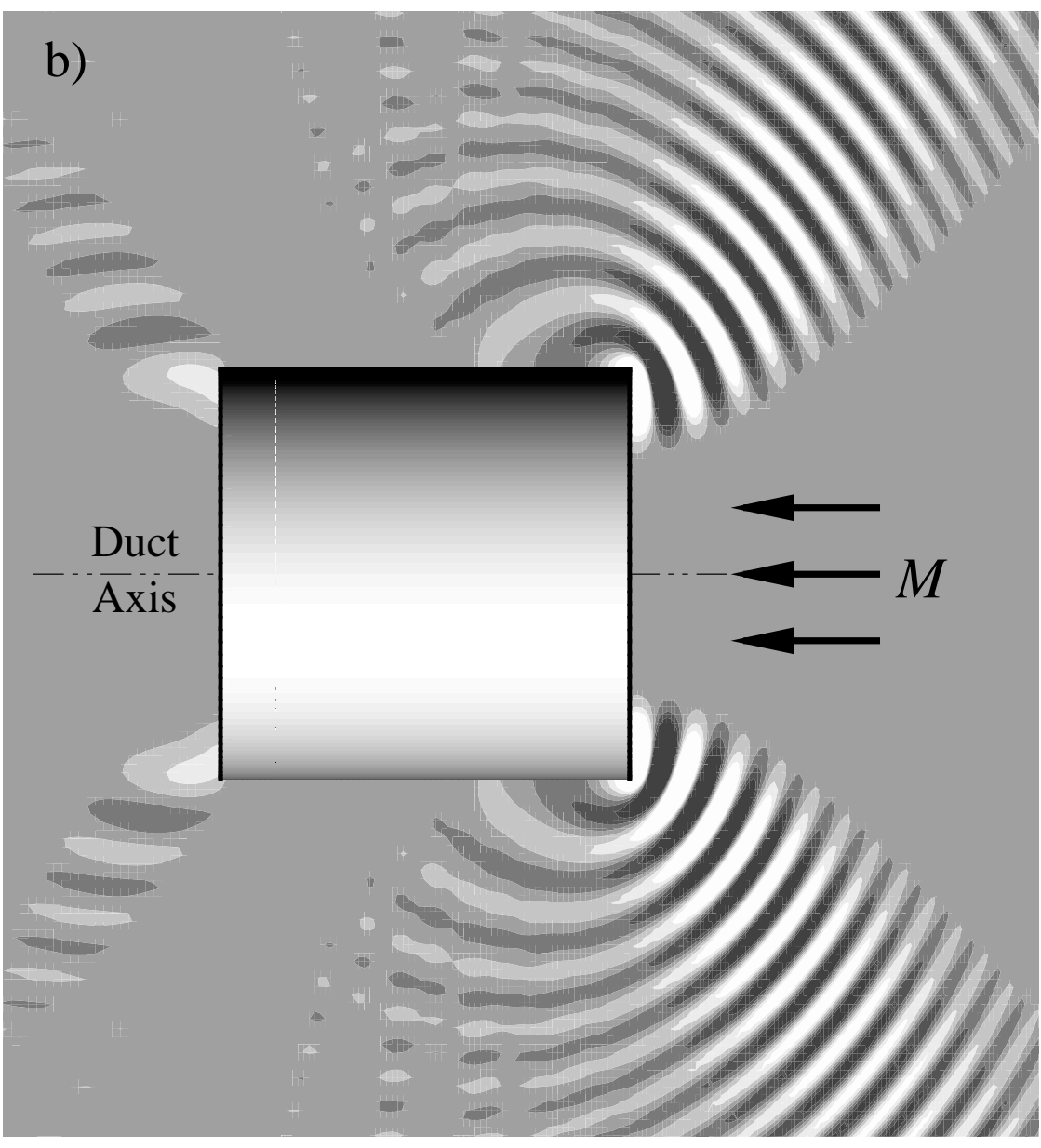

$\mathrm{Re}[\mathrm{P}]$, Pascals

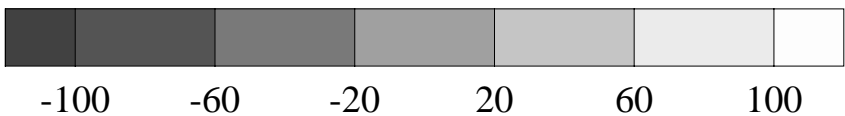

Figure 2: Sample TBIEM3D Results (Hardwall Interior)

Contours of Instantaneous Acoustic Pressure (Pa)

a) Cut-Away View b) Side View

$$
N=20 \quad m=20 \quad k=22 \quad M=0.4 \quad L_{D}=0.5
$$

One Radial Mode Cut-on 
a)

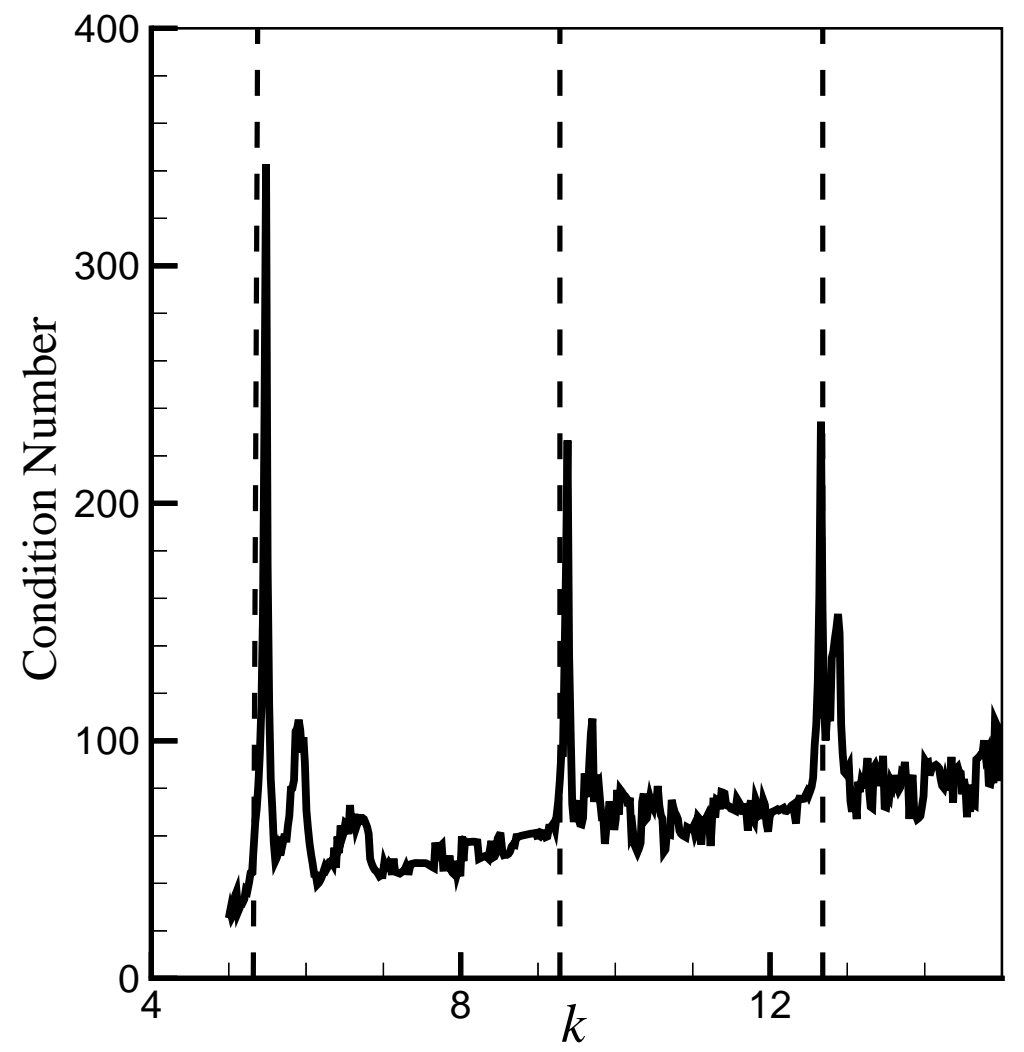

b)

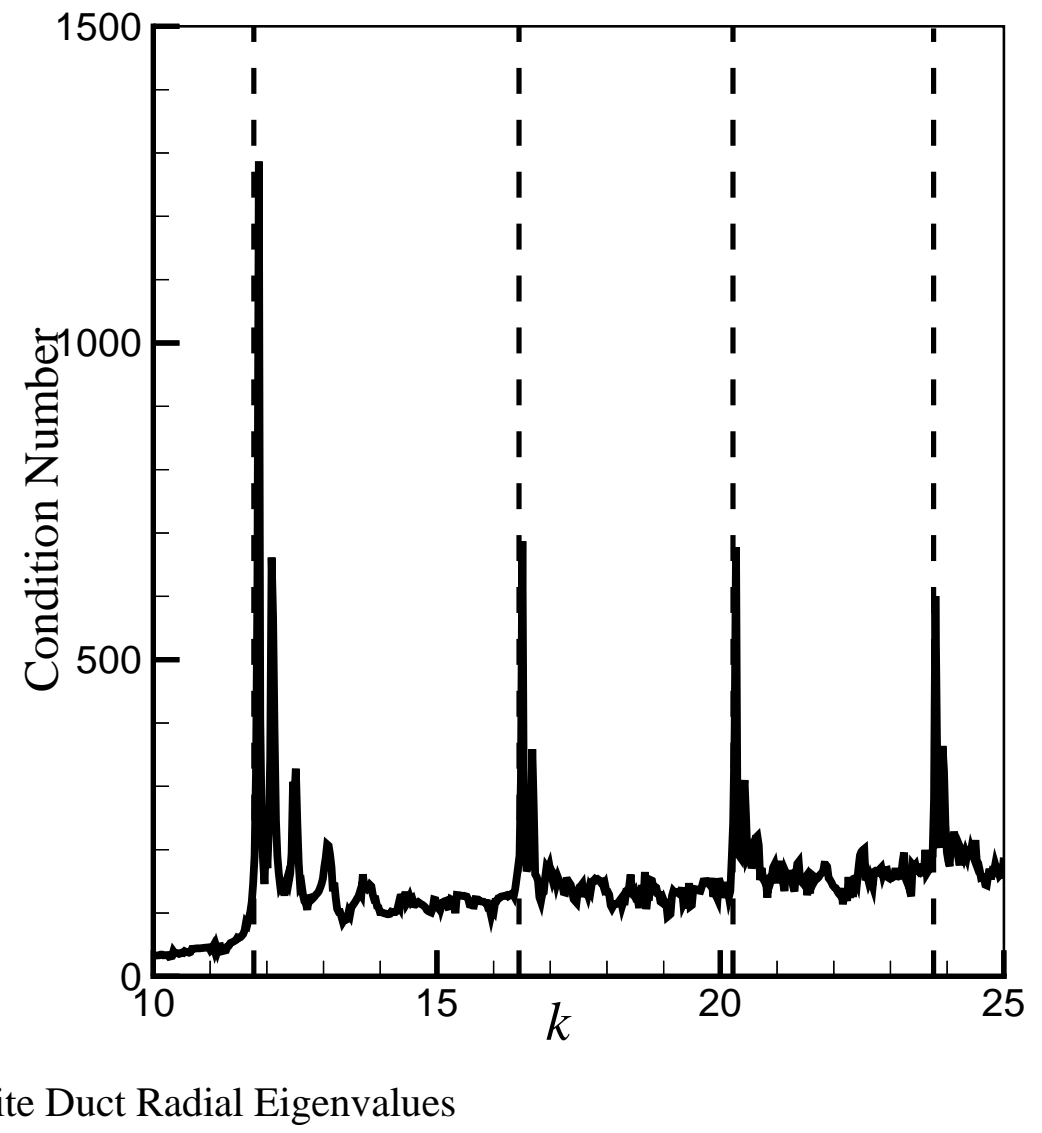

Figure 3: $L_{l}$ Condition Number of $\mathbf{D}_{\mathbf{r}}^{+}$Matrix as a Function of Wavenumber

$$
\begin{array}{cc}
\text { a) } \mathrm{m}=4 & \text { b) } \mathrm{m}=10 \\
M=0.0 & L_{D}=6.0
\end{array}
$$


a)

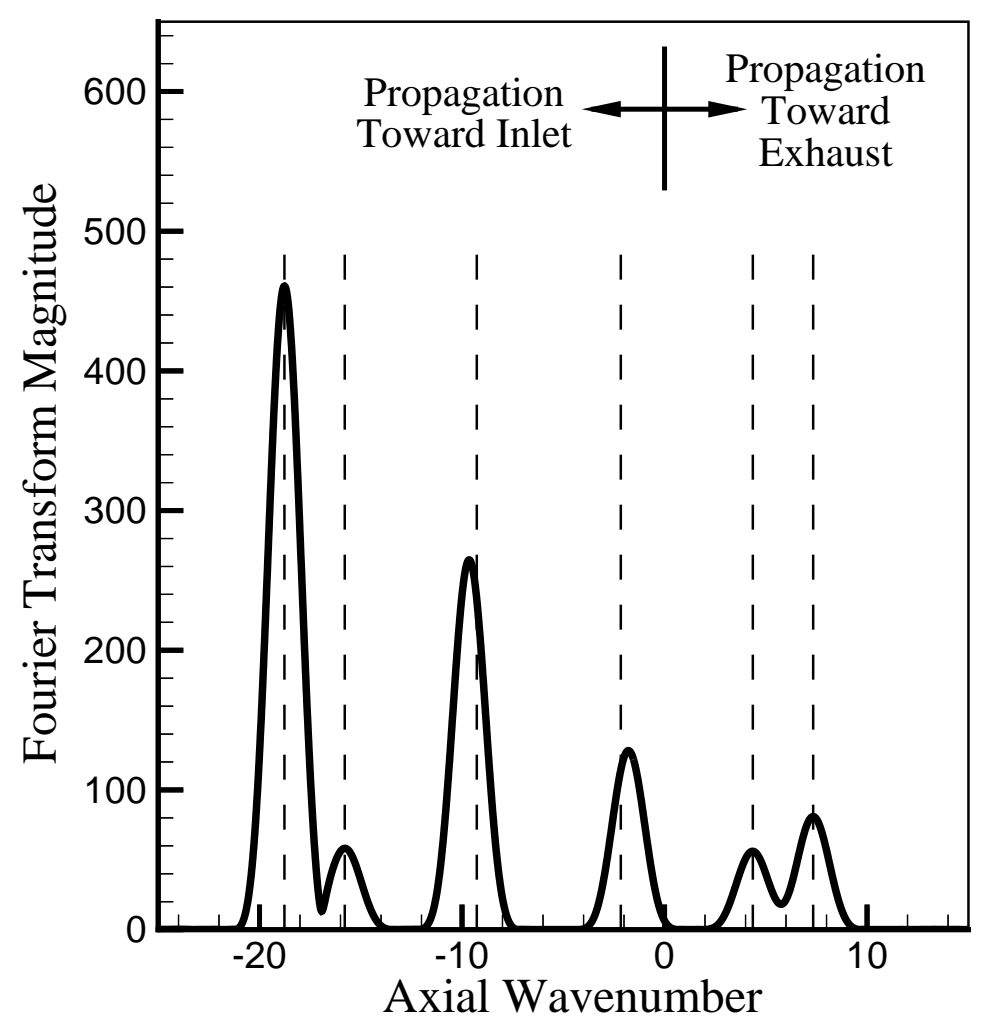

b)

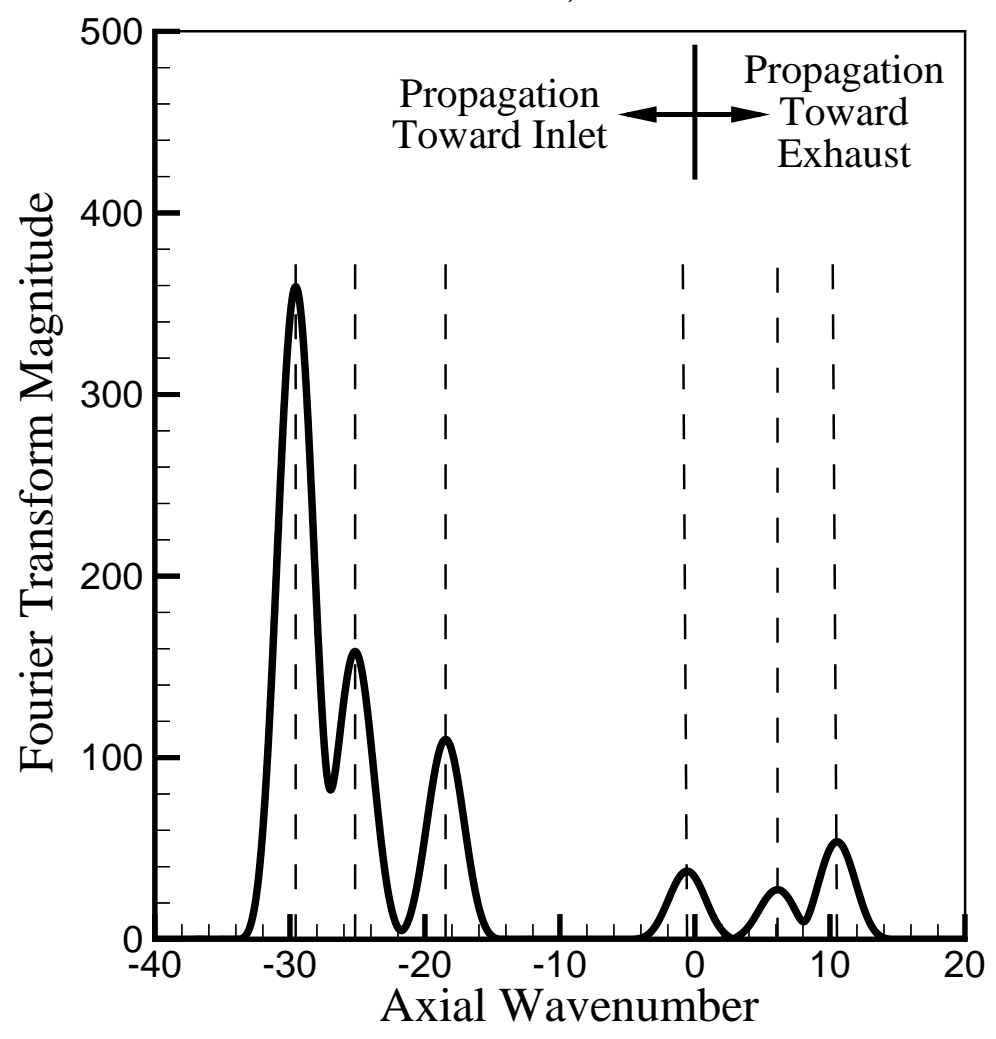

Figure 4: Axial Wavenumber Spectra for Hardwall Interior
a) $\mathrm{m}=4 \mathrm{k}=12.0$
b) $\mathrm{m}=10 \mathrm{k}=20.0$
$M=0.4 \quad L_{D}=6.0$
BIEM
_ _ _ _ Infinite Duct 
a)

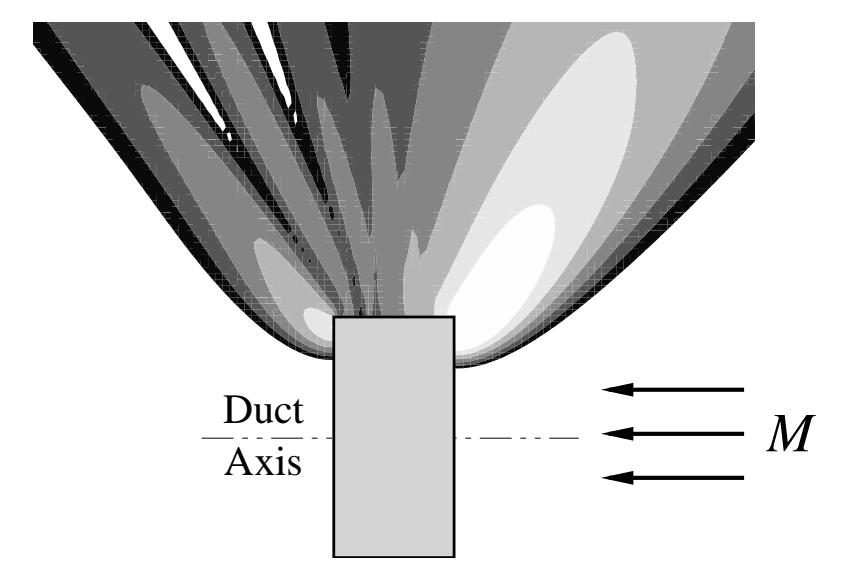

c)

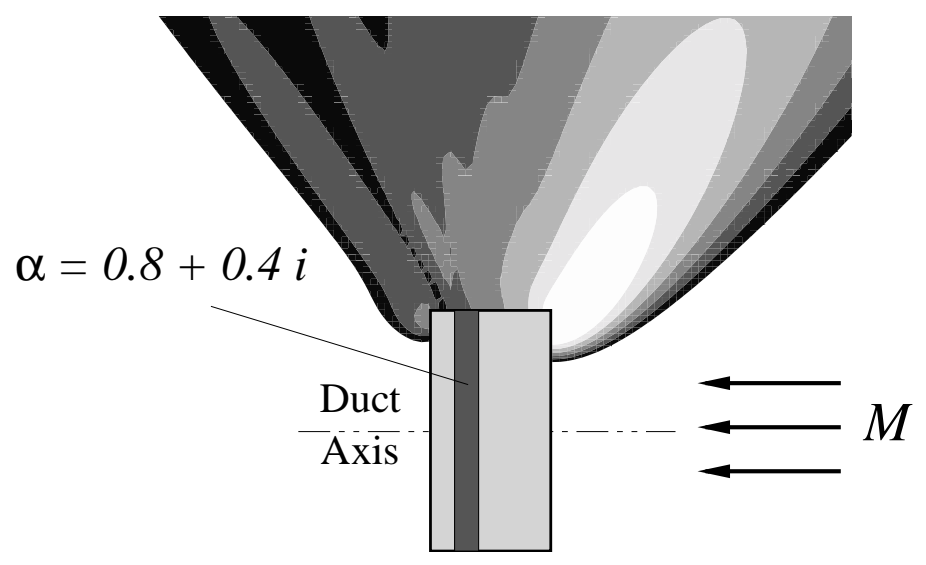

b)

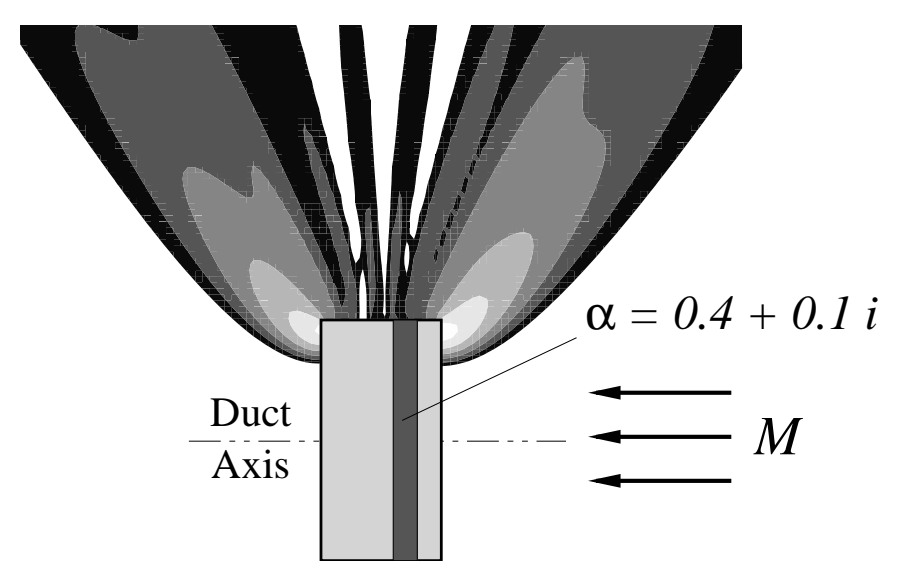

d)

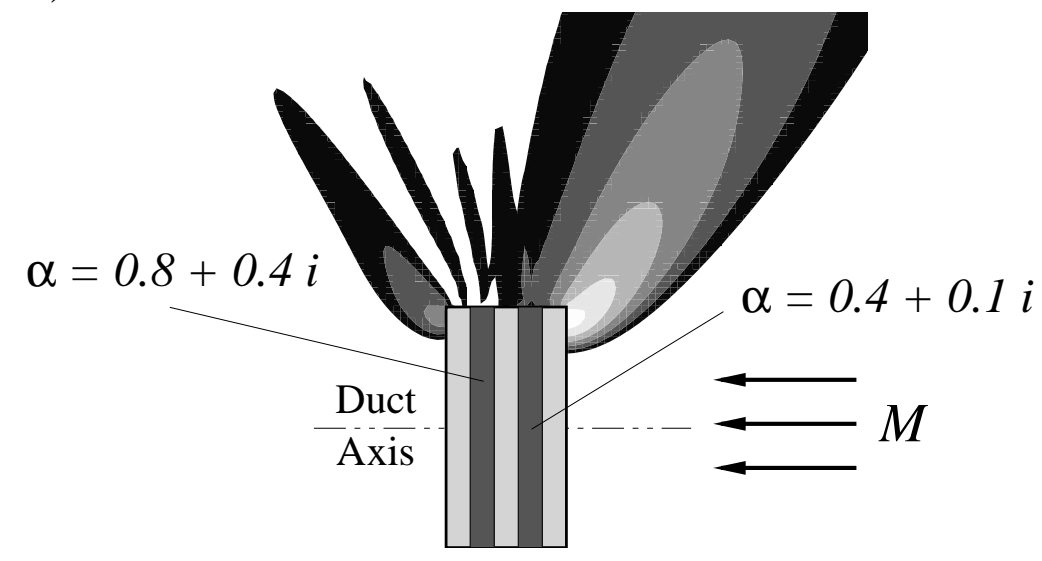

SPL, dB $(\operatorname{Re} 20 \mu \mathrm{Pa})$

$\begin{array}{lllll}120 & 125 & 130 & 135 & 140\end{array}$

Figure 5: Sound Pressure Levels for Various Liner Admittance Distributions

a) Hardwall Interior b) Lined Inlet c) Lined Exhaust d) Lined Inlet and Exhaust $m=20 \quad k=24.0 \quad M=0.4 \quad L_{D}=0.5$ 